Kevin Fritsch (1) · Peter Heinzner(1)

\title{
Equivariant embeddings of strongly pseudoconvex Cauchy-Riemann manifolds
}

Received: 19 May 2020 / Accepted: 13 March 2021 / Published online: 28 March 2021

\begin{abstract}
Let $X$ be a CR manifold with transversal, proper CR action of a Lie group $G$. We show that the quotient $X / G$ is a complex space such that the quotient map is a CR map. Moreover the quotient is universal, i.e. every invariant CR map into a complex manifold factorizes uniquely over a holomorphic map on $X / G$. We then use this result and complex geometry to prove an embedding theorem for (non-compact) strongly pseudoconvex CR manifolds with transversal $G \rtimes S^{1}$-action. The methods of the proof are applied to obtain a projective embedding theorem for compact CR manifolds.
\end{abstract}

\section{Introduction}

An important and much studied question in CR geometry is whether an abstract CR manifold can be realized, locally or even globally, as a CR submanifold of $\mathbb{C}^{n}$, see for example [1,4] or [15]. There have also been several works on the topic of CR manifolds with transversal group actions [2], Lempert proved an embedding result for the otherwise difficult 3-dimensional case assuming the existence of a transversal CR $\mathbb{R}$-action [16].

There have been more recent results for CR manifolds with transversal $S^{1}$ action by Herrmann et al. [12] and also an equivariant Kodaira embedding theorem by Hsiao et al. [13].

Most of the above results are for the case of CR codimension 1, but the high codimension case is also interesting, as the following example from the theory of transformation groups shows.

Let $(Z, \omega)$ be a Kähler manifold with holomorphic action of a Lie group $G$ which leaves $\omega$ invariant. Let $\mu: Z \rightarrow \mathfrak{g}^{*}$ be a momentum map such that 0 is a regular value and define $\mathcal{M}:=\mu^{-1}(0)$. Then $T_{x} \mathcal{M} \cap i T_{x} \mathcal{M}=(\mathbb{C g}(x))^{\perp_{\omega}}$ and $\mathcal{M}$ is a CR submanifold of $Z$ with transversal $G$-action. Here, $\mathfrak{g}(x)$ denotes the tangent space at $x$ to the orbit $G x$ and $\mathbb{C g}(x)$ the complex subspace generated by $\mathfrak{g}(x)$.

The space $\mathcal{M}$ and the induced complex structure on the quotient $\mathcal{M} / G$ is of high interest in geometric invariant theory. In the case where $Z$ is a bounded domain in $\mathbb{C}^{n}$

K. Fritsch (凶): Ruhr-University, Bochum, Germany

e-mail: kevin.fritsch@rub.de

P. Heinzner: e-mail: peter.heinzner@ rub.de

Mathematics Subject Classification: 32V30 - 22E · 57M60

https://doi.org/10.1007/s00229-021-01291-w 
with Bergmann metric $\omega$ and $G$ a unipotent subgroup of the group of holomorphic isometries of $(Z, \omega)$, very little is generally known about the quotient $\mathcal{M} / G$.

In this paper we will consider $X$ to be a CR manifold with proper, transversal action of a group $G$ such that $G$ is a subgroup of its universal complexification $G^{\mathbb{C}}$. We recall that $G$ is a subgroup of its universal complexification if and only if it is a Lie subgroup of some complex Lie group. For example every closed subgroup of a matrix group is a subgroup of a complex group but the universal covering of $S L_{2}(\mathbb{R})$ is not.

We systematically start by showing that $X$ may always be embedded into a complex manifold. In particular, we say that a complex manifold $Z$ with holomorphic action of $G^{\mathbb{C}}$ and a $G$-equivariant $\mathrm{CR}$ embedding $\Phi: X \rightarrow Z$ is the universal equivariant extension of $X$ if every equivariant CR map $f: X \rightarrow Y$ into a complex manifold $Y$ with holomorphic $G^{\mathbb{C}}$-action extends uniquely to a $G^{\mathbb{C}}$-equivariant holomorphic map on $Z$.

Theorem 1.6. Let $X$ be a CR manifold with proper, transversal, CR action of a Lie group $G$. Assume that $G$ is a subgroup of its universal complexification. Then there exists a universal equivariant extension for $X$.

We use this to show that the quotient space $X / G$ carries the structure of a complex space such that the sheaf of holomorphic functions on $X / G$ is given by the sheaf of $G$-invariant CR functions on $X$ (Theorem 1.8). In Sect. 2, we will generalize the notion of strong pseudoconvexity to CR manifolds with transversal of codimension one group action. We will then prove a connection between strongly pseudoconvex CR manifolds and $S^{1}$-bundles in positive orbifold line bundles (see Theorem 2.3).

Using the quotient result and methods from complex geometry, we prove the following equivariant embedding theorem. Let $H$ be a closed subgroup of its universal complexification, such that $H^{\mathbb{C}}$ is complex reductive and $H=G \rtimes S^{1}$, then we have $H^{\mathbb{C}}=G^{\mathbb{C}} \rtimes \mathbb{C}^{*}$. Let $X$ be a CR manifold with proper, transversal, CR action of $H$ such that $H_{x}^{0}<G_{x}^{0}$ for every $x \in X$ and let $Y$ be the universal equivariant exension of $X$.

Theorem 3.5. Under the assumptions above, let $X$ be strongly pseudoconvex and $X / G$ be compact. Then there exists a $H^{\mathbb{C}}$-representation $V$ and $a H^{\mathbb{C}}$-equivariant holomorphic embedding $\Phi: Y \rightarrow \mathbb{C}^{m} \backslash\{0\} \times V$, such that $\left.\Phi\right|_{X}: X \rightarrow \mathbb{C}^{m} \times V$ is an embedding. Here, $\mathbb{C}^{m}$ is the trivial $G^{\mathbb{C}}$-representation and decomposes into irreducible $\mathbb{C}^{*}$-representations with positive weights.

In chapter 4, we use these techniques for a proof of a Kodaira-type embedding theorem for CR manifolds.

Corollary 4.1. Let $X$ be a compact $C R$ manifold with a transversal CR action of a compact Lie group $K$. Assume that there exists a weakly negative line bundle $L_{K} \rightarrow X / K$ and let $L \rightarrow X$ be the induced $C R$ line bundle. Then there exists a natural number $k$ and finitely many $C R$ sections $s_{i} \in \Gamma\left(X, L^{-k}\right)$ such that, for $W=\operatorname{span}\left(s_{i}\right)$, we have that

$$
\begin{aligned}
X & \rightarrow \mathbb{P}\left(W^{*}\right) \\
y & \mapsto[s \mapsto s(y)]
\end{aligned}
$$


is a CR embedding.

Moreover we will show that the above embedding can be chosen to be $K$ equivariant.

\section{Quotients}

Let $X$ be a smooth manifold and $G$ a Lie group with smooth action on $X$. For an element $\xi \in \mathfrak{g}$, we denote by $\xi_{X}(x):=\left.\frac{d}{d t}\right|_{0} \exp (t \xi) x$ the fundamental vector field of $\xi$ on $X$. We set $\mathfrak{g}(x):=\left\{\xi_{X}(x) \mid \xi \in \mathfrak{g}\right\}$ and say that the action is locally free if $\xi_{X}(x) \neq 0$ for every $\xi \in \mathfrak{g} \backslash\{0\}$.

For a manifold $X$, we write $\mathbb{C} T X$ for the complexified tangent bundle.

Let $T^{1,0} X$ be a smooth complex subbundle of $\mathbb{C} T X$ such that $T^{1,0} X \cap \overline{T^{1,0} X}=$ $\{0\}$ and $\left[\Gamma\left(U, T^{1,0} X\right), \Gamma\left(U, T^{1,0} X\right)\right] \subset \Gamma\left(U, T^{1,0} X\right)$ for every open subset $U$ of $X$. Here, $\Gamma\left(U, T^{1,0} X\right)$ denote the smooth sections into $T^{1,0} X$ on $U$. Set $n=\operatorname{dim}_{\mathbb{C}} T^{1,0} X$ and $d=\operatorname{dim}_{\mathbb{R}} X-2 n$, then we call $\left(X, T^{1,0} X\right)$ a CR manifold of dimension $(2 n, d)$. We write $T^{0,1} X:=\overline{T^{1,0} X}$.

A typical example is given by a real submanifold $X$ of a complex submanifold $Z$ such that $\operatorname{dim}\left(T_{x} X \cap J T_{x} X\right)$ is constant in $x$, where $J$ denotes the complex structure on $Z$.

Let $\left(X, T^{1,0} X\right)$ be a CR manifold with a CR action of a Lie group $G$. We call the action transversal, if $\mathbb{C g}(x) \oplus T_{x}^{1,0} X \oplus T_{x}^{0,1} X=\mathbb{C} T_{x} X$ for every $x \in X$, where $\mathbb{C} \mathfrak{g}(x)$ is the complex subspace in $\mathbb{C} T_{x} X$ generated by $\mathfrak{g}(x)$.

A result of Loose states that if the action fulfills $\mathbb{C g}(x) \cap T_{x}^{1,0} X \oplus T_{x}^{0,1} X=\{0\}$ and is proper and free, then the quotient $X / G$ may be equipped with a CR structure such that the projection $X \rightarrow X / G$ is a CR map [17, Theorem 1.1]. Our first goal is to show that if the action is proper and transversal, but not necessarily free, then the quotient $X / G$ is a complex space.

For the proof, we first recall some basic techniques regarding quotients in the smooth case.

Let $X$ be a smooth manifold and $G$ a Lie group with proper and free action on $X$. For every point $x \in X$, we find a smooth submanifold $S$ of $X$ with $x \in S$ such that the map $G \times S \rightarrow X,(g, s) \mapsto g s$ is a diffeomorphism onto an open subset $U$ of $X$ [20, Theorem 2.3.3]. The quotient map $\pi: X \rightarrow X / G$ induces a homeomorphism $\left.\pi\right|_{S}: S \rightarrow \pi(U)$, which defined on $X / G$ the structure of a smooth manifold.

If $G$ is a closed subgroup of a Lie group $H$ and $G$ acts on a manifold $X$, then $G$ acts proper and free on $H \times X$ via $(g,(h, x)) \mapsto\left(h g^{-1}, g x\right)$, hence $H \times{ }^{G} X:=$ $(H \times X) / G$ is a manifold.

This lets us formulate the general slice theorem as follows. Let $G$ act on $X$ properly, then around every $x \in X$, there exists a slice, i.e. a smooth, $G_{X}$-invariant submanifold $S$ of $X$ such that the map $G \times{ }^{G_{x}} S \rightarrow X,[g, s] \mapsto g s$ is a diffeomorphism onto an open subset of $X$ [20, Theorem 2.3.3]. 
On CR manifolds, it is in general not possible to construct a reasonable CR structure on the quotient using slices directly. More precisely, if $G$ acts freely and transversally on a CR manifold $X$ and $S$ is a slice at $x \in X$ such that $\mathbb{C} T_{x} S=T_{x}^{1,0} X \oplus T_{x}^{0,1} X$, then this would imply that $T^{1,0} X \oplus T^{0,1} X$ is involutive and $X$ is flat. This fails in general.

We will use the following Lemma to construct complex structures on quotient spaces. For a smooth map $f: X \rightarrow Y$ between manifolds, we may extend $d f$ to a $\mathbb{C}$-linear map $d f: \mathbb{C} T X \rightarrow \mathbb{C} T Y$. For the sake of simplicity, we will denote the extension with $d f$, as well.

Lemma 1.1. Let $X, Y$ be smooth manifolds and $\pi: X \rightarrow Y$ a surjective submersion. Let $E$ be a smooth, complex subbundle of $\mathbb{C} T X$. For every $y \in Y$, let $F_{y}$ be a complex subspace of $\mathbb{C} T_{y} Y$ such that $d_{x} \pi: E_{x} \rightarrow F_{\pi(x)}$ is an isomorphism for every $x \in X$. Then $F=\bigcup_{y} F_{y}$ is a smooth subbundle of $\mathbb{C} T Y$ and for every $x \in X$, there exist open neighborhoods $U$ of $x$ and $\Omega$ of $\pi(x)$ with $\pi(U)=\Omega$, such that for every smooth section $W \in \Gamma(\Omega, F)$, there exists a smooth, $\pi$-related section $V \in \Gamma(U, E)$, i.e. we have $d \pi \circ V=W \circ \pi$.

Proof. Denote by $B^{n}$ the ball with radius 1 in $\mathbb{R}^{n}$.

Since the result is local in $X$, we may assume that $X=B^{n} \times B^{d}, Y=B^{n}$ and $\pi$ is the projection onto the first component. We may also assume that we find smooth sections $V_{i} \in \Gamma(X, E), i=1, . ., k$, such that $V_{i}(p)$ form a complex basis for $E_{p}$ in every point $p \in X$.

Now fix $w \in B^{d}$, set $s_{w}: B^{n} \rightarrow B^{n} \times B^{d}, z \mapsto(z, w)$ and define $W_{i}^{w}(z):=d_{(z, w)} \pi\left(V_{i}\left(s_{w}(z)\right)\right)$. Since $d \pi: E \rightarrow F$ is an isomorphism in every point, the $W_{i}^{w}(z)$ define a basis for $F_{w}$ for every $w \in B^{d}$ and $z \in B^{n}$ which depends smoothly on $w$ and $z$. In particular, the space $F=\bigcup_{w} F_{w}$ defines a smooth subbundle of $\mathbb{C} T Y$.

Now let $W \in \Gamma(Y, F)$ be a smooth section. For $w \in B^{d}$, we write $W(z)=$ $\sum_{i} f_{i}^{w}(z) W_{i}^{w}(z)$ for complex-valued smooth functions $f_{i}^{w}$.

Then the $\pi$-related smooth section is given by $V(z, w):=\sum_{i} f_{i}^{w}(z) V_{i}(z, w)$.

We formalize the notion of CR embeddings.

Definition. Let $X, Y$ be CR manifolds. A map $\Phi: X \rightarrow Y$ is called a CR embedding if it is a smooth embedding of $X$ into $Y$ and

$$
d \Phi\left(T^{1,0} X\right)=d \Phi(\mathbb{C} T X) \cap T^{1,0} Y,
$$

i.e. $\Phi(X)$ is a CR submanifold of $Y$. We require all embeddings to be closed.

Note that in the case where $X$ is of CR codimension 1, it suffices that $\Phi$ is an embedding and a CR map. For higher CR codimension however, this is not true. 
If a CR manifold $X$ may be embedded into a complex manifold $Z$, it is an interesting question whether the CR functions on $X$ may (locally) be extended to holomorphic functions on $Z$.

If $H$ is a complex Lie group acting on a complex manifold $Z$, we say that the action is holomorphic if the action map $H \times Z \rightarrow Z$ is holomorphic.

Definition. Let $G$ be a Lie group and $X$ a CR manifold with transversal action of $G$. Let $Z$ be a complex manifold with holomorphic $G^{\mathbb{C}}$-action and $\Phi: X \rightarrow Z$ a $G$-equivariant $\mathrm{CR}$ map. We say that $(Z, \Phi)$ is a universal equivariant extension of $X$ if for every complex manifold $Y$ with holomorphic $G^{\mathbb{C}}$-action and every $G$ equivariant CR map $f: X \rightarrow Y$, there exists a unique $G^{\mathbb{C}}$-equivariant holomorphic map $F: Z \rightarrow Y$ with $f=F \circ \Phi$.

If the universal equivariant extension exists, it is unique up to a $G^{\mathbb{C}}$-equivariant biholomorphic map.

From now on, denote by $G^{\mathbb{C}}$ the universal complexification of $G$ and assume that $G$ is a subgroup of $G^{\mathbb{C}}$. This is for example the case if there exists any injective morphism of $G$ into a complex Lie group. In particular, it is the case for every linear group. Now $G$ being a subgroup of $G^{\mathbb{C}}$ implies that $G$ is a totally real, closed subgroup of $G^{\mathbb{C}}$ [11, 1 Proposition].

Let $X$ be a CR manifold with proper, transversal CR action of $G$. For a Lie group $G$, we will denote by $G^{0}$ the connected component of the identity, which is a normal Lie subgroup of $G$.

Let $S$ be a (smooth) slice at $x \in X$ and $L=G_{x}$. Because all $G$-orbits are of the same dimension, all isotropy groups $G_{s}$ for $s \in S$ have to contain $L^{0}$. Since $L^{0}$ acts trivially on $S$, we get an $L / L^{0}$-action on $S$ and the $L$-orbits on $S$ are finite.

Since $L$ is compact, we have $L^{\mathbb{C}}=L \exp (i \mathfrak{l})$ and every connected component of $L^{\mathbb{C}}$ intersects $L$. We conclude that $L^{\mathbb{C}} /\left(L^{0}\right)^{\mathbb{C}}=L / L^{0}$ and get an $L^{\mathbb{C}}$-action on $S$ as a finite group.

Because of [11, 3 Corollary 1], we see that $L^{\mathbb{C}}$ is a closed complex subgroup of $G^{\mathbb{C}}$. For $\Omega=G \times{ }^{L} S$, define the smooth manifold

$$
\Omega^{\mathbb{C}}:=G^{\mathbb{C}} \times{ }^{L^{\mathbb{C}}} S .
$$

Note that for $s \in S$, we get $\left(L_{s}\right)^{\mathbb{C}}=\left(L^{\mathbb{C}}\right)_{s}$. Hence for $g \in G$ and $[g, s] \in$ $G^{\mathbb{C}} \times L^{\mathbb{C}} S$, we conclude $\left(G^{\mathbb{C}}\right)_{[g, s]}=g\left(L^{\mathbb{C}}\right)_{s} g^{-1}=g\left(L_{S}\right)^{\mathbb{C}} g^{-1}=\left(G_{[g, s]}\right)^{\mathbb{C}}$.

We call $\Omega^{\mathbb{C}}$ the extension of the slice $S$ or just a slice extension around $x$.

We want to show that $\Omega$ may be embedded into $\Omega^{\mathbb{C}}$ and start with the following Lemma.

Lemma 1.2. Let $G$ be a closed subgroup of $H$. Let $H_{0}$ be a closed subgroup of $H$, define the subgroup $G_{0}:=G \cap H_{0}$ and assume that the image of the map $G / G_{0} \rightarrow H / H_{0}$ is closed. Let $X$ be a smooth manifold with $H_{0}$-action, then the map $\Phi: G \times{ }^{G_{0}} X \rightarrow H \times{ }^{H_{0}} X$ is an embedding.

Proof. By construction of $G_{0}$ and $H_{0}$, the map $G / G_{0} \rightarrow H / H_{0}$ is an immersion. In particular, it is a smooth embedding [19, 2.13 Theorem]. Since the following diagram of bundle maps 


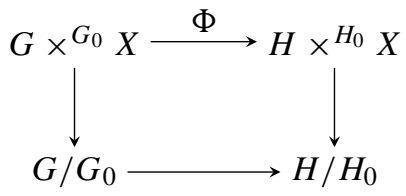

commutes, it follows that $\Phi$ is an embedding.

Lemma 1.3. The natural map $\Phi: G \times{ }^{L} S \rightarrow G^{\mathbb{C}} \times{ }^{L^{\mathbb{C}}} S$ is an embedding.

Proof. The idea of the proof is to apply Lemma 1.2 for $H=G^{\mathbb{C}}, H_{0}=L^{\mathbb{C}}$ and $H=L$. Due to [11, 3 Corollary 1], we have $L^{\mathbb{C}} \cap G=L$. It remains to show that the image of $G / L \rightarrow G^{\mathbb{C}} / L^{\mathbb{C}}$ is closed.

From [11, 1 Proposition] we get the existence of an involutive anti-holomorphic homomorphism $\Theta$ on $G^{\mathbb{C}}$ such that $G$ is the fixed point set of $\Theta$.

Let $\theta$ be the corresponding involution on $\mathfrak{g}^{\mathbb{C}}$, then $\mathfrak{l}$ is fixed under $\theta$ and $\mathfrak{l}^{\mathbb{C}}$ is invariant. Since $L$ is compact, we have $L^{\mathbb{C}}=L \exp (i l)$.

Now let $g_{n}$ be a sequence in $G$ such that the image of $g_{n}$ in $G^{\mathbb{C}} / L^{\mathbb{C}}$ converges. We find a sequence $l_{n} \in L^{\mathbb{C}}$ such that $g_{n} l_{n} \rightarrow g \in G^{\mathbb{C}}$.

Write $l_{n}=k_{n} \exp \left(P_{n}\right)$ with $k_{n} \in L$ and $P_{n} \in i$ l. Then

$$
\begin{aligned}
& l_{n} g_{n}^{-1} \Theta\left(l_{n} g_{n}^{-1}\right)^{-1}=l_{n} \Theta\left(l_{n}\right)^{-1}=k_{n} \exp \left(P_{n}\right) \Theta\left(k_{n} \exp \left(P_{n}\right)\right)^{-1} \\
& =k_{n} \exp \left(P_{n}\right) \exp \left(-\theta\left(P_{n}\right)\right) k_{n}^{-1}=k_{n} \exp \left(2 P_{n}\right) k_{n}^{-1},
\end{aligned}
$$

using the same computation as above. Since $L$ is compact, we may assume that $k_{n}$ and therefore $\exp \left(P_{n}\right)$ converges. This implies that $l_{n}$ and therefore $g_{n}$ is a convergent sequence.

In order to formulate our first result, we fix $x \in X$ and a Slice $S$ at $x$. We identify $\Omega=G \cdot S$ with $G \times{ }^{L} S$ where $L=G_{x}$ and denote by $\Omega^{\mathbb{C}}=G^{\mathbb{C}} \times{ }^{L^{\mathbb{C}}} S$ the corresponding slice extension. We have the following

Theorem 1.4. (1) The slice extension $\Omega^{\mathbb{C}}$ is a complex manifold such that the natural $G^{\mathbb{C}}$-action on $\Omega^{\mathbb{C}}$ is holomorphic.

(2) The G-equivariant map $\Phi: G \times^{L} S \rightarrow G^{\mathbb{C}} \times^{L^{\mathbb{C}}} S$ is a CR embedding.

(3) For every $G$-invariant open subset $U \subset \Omega$, the restriction $\left.\Phi\right|_{U}: U \rightarrow G^{\mathbb{C}} U$ is a CR embedding and $G^{\mathbb{C}} U$ is a universal complexification of $U$.

Proof. We begin by showing that $\Omega^{\mathbb{C}}$ is a complex manifold. The map

$$
\begin{gathered}
\eta: G^{\mathbb{C}} \times \Omega \rightarrow \Omega^{\mathbb{C}} \\
(h, x) \mapsto h \Phi(x) .
\end{gathered}
$$

is a surjective submersion. We denote by $[h, s]_{C}$ the elements of $G^{\mathbb{C}} \times{ }^{L^{\mathbb{C}}} S$.

The fiber $\eta^{-1}\left(\left[1, s_{0}\right]_{C}\right)$ consists of the elements $(h,[g, s]) \in G^{\mathbb{C}} \times\left(G \times{ }^{L} S\right)$ such that $[h g, s]_{C}=\left[1, s_{0}\right]_{C}$. In particular, this means that there exists an $l \in L^{\mathbb{C}}$ such that $l s=s_{0}$. But since the $L^{\mathbb{C}}$-orbits on $S$ are equal to the $L$-orbits, we may choose $l$ to be in $L$. We get

$$
\eta^{-1}\left(\left[1, s_{0}\right]_{C}\right)=\left\{\left(h,\left[g, s_{0}\right]\right) \mid\left[h g, s_{0}\right]_{C}=\left[1, s_{0}\right]_{C}\right\} .
$$


This condition is equivalent to the existence of an $l \in\left(G^{\mathbb{C}}\right)_{\left[1, s_{0}\right]}=\left(G_{\left[1, s_{0}\right]}\right)^{\mathbb{C}}$ such that $h g l^{-1}=1$, hence $h=l g^{-1}$. We have shown that

$$
\eta^{-1}\left(\left[1, s_{0}\right]_{C}\right)=\left\{\left(l g^{-1},\left[g, s_{0}\right]\right) \mid l \in\left(G_{\left[1, s_{0}\right]}\right)^{\mathbb{C}}, g \in G\right\} .
$$

Now let $h_{0} \in G^{\mathbb{C}}$. Since $\eta$ is $G^{\mathbb{C}}$-equivariant, we get

$$
\eta^{-1}\left(\left[h_{0}, s_{0}\right]\right)=\left\{\left(h_{0} l g^{-1},\left[g, s_{0}\right]\right) \mid l \in\left(G_{\left[1, s_{0}\right]}\right)^{\mathbb{C}}, g \in G\right\} .
$$

Since $\eta$ is a submersion, every fiber of $\eta$ is a submanifold with tangent space equal to the kernel of $d \eta$. hence in a point $\left(h_{0}, y\right) \in G^{\mathbb{C}} \times \Omega$, we get

$$
\operatorname{ker} d_{\left(h_{0}, y\right)} \eta=\left\{\left(d h_{0}(\xi-\mu), \mu_{\Omega}(y)\right) \mid \xi \in\left(\mathfrak{g}_{y}\right)^{\mathbb{C}}, \mu \in \mathfrak{g}\right\},
$$

where $\left(\mathfrak{g}_{y}\right)^{\mathbb{C}}$ denotes the Lie algebra of the isotropy group $\left(G^{\mathbb{C}}\right)_{y}$ and $d h_{0}$ is the differential of the left translation by $h_{0}$.

Now we consider $G^{\mathbb{C}} \times \Omega$ as a CR manifold. Let $W \in \operatorname{ker} d_{\left(h_{0}, y\right)} \eta \cap T^{1,0}\left(G^{\mathbb{C}} \times\right.$ $\Omega)$. Since the $G$-action on $\Omega$ is transversal, we have $W=\left(d h_{0}(\xi), 0\right)$ for $\xi \in$ $\mathbb{C}\left(\mathfrak{g}_{y}\right)^{\mathbb{C}}$. Define

$$
K_{\left(h_{0}, y\right)}:=\operatorname{ker} d_{\left(h_{0}, y\right)} \eta \cap T_{\left(h_{0}, y\right)}^{1,0}\left(G^{\mathbb{C}} \times \Omega\right)=\left\{\left(d h_{0}(\xi), 0\right) \mid \xi \in T_{1}^{1,0}\left(G_{y}\right)^{\mathbb{C}}\right\}
$$

then

$$
\bar{K}_{\left(h_{0}, y\right)}=\left\{\left(d h_{0}(\xi), 0\right) \mid \xi \in T_{1}^{0,1}\left(G_{y}\right)^{\mathbb{C}}\right\}
$$

and

$$
\operatorname{ker} d_{\left(h_{0}, y\right)} \eta \cap\left(T^{1,0}\left(G^{\mathbb{C}} \times \Omega\right) \oplus T^{0,1}\left(G^{\mathbb{C}} \times \Omega\right)\right)=K_{\left(h_{0}, y\right)} \oplus \bar{K}_{\left(h_{0}, y\right)} .
$$

Since all isotropy groups of the $G$-action on $\Omega$ are of the same dimension, we see that the dimension of $K_{y}$ does not depend on $y \in G^{\mathbb{C}} \times \Omega$, hence it defines a complex subbundle of $T^{1,0}\left(G^{\mathbb{C}} \times \Omega\right)$. Let $E$ be a complex subbundle of $T^{1,0}\left(G^{\mathbb{C}} \times \Omega\right)$ such that $E \oplus K=T^{1,0}\left(G^{\mathbb{C}} \times \Omega\right)$.

For $x \in G^{\mathbb{C}} \times \Omega$, define $F_{x}:=\left\{d_{x} \eta(V) \mid V \in T_{x}^{1,0}\left(G^{\mathbb{C}} \times \Omega\right)\right\}$ and for $y \in \Omega^{\mathbb{C}}$, set $F_{y}:=\bigcup_{\eta(x)=y} F_{x}$. Note that $d \eta$ defines an isomorphism between $E_{x}$ and $F_{x}$ in every point. We want to show that $F_{y}=F_{x}$ for every $x \in \eta^{-1}(y)$.

The map $\eta$ is invariant under the $\mathrm{CR}$ action of $G$ on $G^{\mathbb{C}} \times \Omega$ via $(g,(h, x)) \mapsto$ $\left(h g^{-1}, g x\right)$, which shows that $F_{[h, g x]}=F_{[h g, x]}$ for $g \in G$.

Because of that, it suffices to show $F_{(h,[1, s])}=F_{\left(h_{0},\left[1, s_{0}\right]\right)}$ if $\eta(h,[1, s])=$ $\eta\left(h_{0},\left[1, s_{0}\right]\right)$. But the latter implies that $[h, s]_{C}=\left[h_{0}, s_{0}\right]_{C}$, hence there exists an $l \in L^{\mathbb{C}}$ such that $l s=s_{0}$ and we may again choose $l$ to be in $L$.

We may therefore assume $s=s_{0}$ and $h h_{0}^{-1} \in\left(G_{[1, s]}\right)^{\mathbb{C}}$. It then remains to prove $F_{(l,[1, s])}=F_{(1,[1, s])}$ for all $l \in\left(G_{[1, s]}\right) \mathbb{C}$.

We have $F_{(l,[1, s])}=F_{[1, l[1, s]]}=F_{(1,[1, s])}$ for all $l \in G_{[1, s]}$. Note that $[1, s] \in \Omega$ implies $\left(G_{[1, s]}\right)^{\mathbb{C}}=\left(G^{\mathbb{C}}\right)_{[1, s]}$.

Because $\eta$ is equivariant, we conclude $F_{(l,[1, s])}=d l\left(F_{(1,[1, s])}\right)$ and need to show that $d l\left(F_{(1,[1, s])}\right)=F_{(1,[1, s])}$ for $l \in G_{[1, s]}^{\mathbb{C}}$. But now $\mathbb{C} T_{\eta(1,[1, s])} \Omega^{\mathbb{C}}$ is a 
holomorphic $G_{[1, s]}^{\mathbb{C}}$-representation and $F_{(1,[1, s])}$ is a complex subspace which is invariant under $G_{[1, s]}$. Therefore it is invariant under $G_{[1, s]}^{\mathbb{C}}$.

We have shown that $d_{x} \eta$ induces an isomorphism between $E_{x}$ and $F_{\eta(x)}=F_{x}$ and from Lemma 1.1 we get that $F=\bigcup_{x} F_{\eta(x)}$ is a subbundle of $\mathbb{C} T \Omega^{\mathbb{C}}$. We claim that $F$ defines a $\mathrm{CR}$ structure on $\Omega^{\mathbb{C}}$.

Let $d_{x} \eta(V), d_{x} \eta(W) \in F_{\eta(x)}$ and assume $d_{x} \eta(V)=\overline{d_{x} \eta(W)}=d_{x} \eta(\bar{W})$. We conclude $V-\bar{W} \in K_{x} \oplus \overline{K_{x}}$ and get $V-\bar{W}=V_{0}-\bar{W}_{0}$ with $V_{0} \in K_{x}$ and $\bar{W}_{0} \in \bar{K}_{x}$. But then $V_{0}=V, W_{0}=W$ and $d_{x} \eta(V)=d_{x} \eta(\bar{W})=0$.

For $V \in \Gamma^{\infty}\left(\Omega^{\mathbb{C}}, F\right)$, we use Lemma 1.1 and find a smooth section $V_{0} \in$ $\Gamma^{\infty}\left(G^{\mathbb{C}} \times \Omega, E\right)$ with $d_{x} \eta V_{0}=V(\pi(x))$. Then

$$
V(\eta(x))(f)=d f(V(\eta(x)))=d f\left(d \eta\left(V_{0}(x)\right)\right)=V_{0}(x)(f \circ \eta),
$$

or alternatively $(V f) \circ \eta=V_{0}(f \circ \eta)$. For another $W \in \Gamma^{\infty}\left(\Omega^{\mathbb{C}}, F\right)$ and $W_{0}$ as above, this then implies

$$
\begin{aligned}
(\eta(x))(f) & =V(W(f))(\eta(x))-W(V(f))(\eta(x)) \\
& =V_{0}\left(W_{0}(f \circ \eta)\right)(x)-W_{0}\left(V_{0}(f \circ \eta)\right)(x) \\
& =\left[V_{0}, W_{0}\right](x)(f \circ \eta)=d \eta\left(\left[V_{0}, W_{0}\right](x)\right)(f) .
\end{aligned}
$$

Hence $[V, W](\eta(x))=d \eta\left(\left[V_{0}, W_{0}\right](x)\right)$ and $[V, W](\eta(x)) \in F_{\eta(x)}$.

We have shown that $\Omega^{\mathbb{C}}$ is a CR manifold. Since its real codimension is zero, it is a complex manifold.

The $G^{\mathbb{C}}$-action on $\Omega^{\mathbb{C}}$ is holomorphic if the pulled back map $G^{\mathbb{C}} \times G^{\mathbb{C}} \times \Omega \rightarrow$ $\Omega^{\mathbb{C}},(g,(h, x)) \mapsto g h \Phi(x)$ is CR. This is true because $\eta$ is a CR map by construction.

Lemma 1.3 says that $\Phi$ is a smooth embedding, let us check that it is a CR embedding. Note that $\Phi$ is a CR map by construction and $\Phi_{0}: \Omega \rightarrow G^{\mathbb{C}} \times \Omega$, $x \mapsto(1, x)$ is a CR embedding with $\Phi=\eta \circ \Phi_{0}$.

Let $y \in \Omega$ and $W \in d_{y} \Phi\left(\mathbb{C} T_{y} \Omega\right) \cap T_{\eta(1, y)}^{1,0} \Omega^{\mathbb{C}}$. By construction of the CR structure, $W=d_{(1, y)} \eta\left(W_{0}\right)$ for $W_{0} \in T_{(1, y)}^{1,0}\left(G^{\mathbb{C}} \times \Omega\right)$ and $W=d_{y} \Phi\left(V_{0}\right)$ for $V_{0} \in \mathbb{C} T_{y} \Omega$.

Now $W_{0}-d_{y} \Phi_{0}\left(V_{0}\right) \in \operatorname{ker} d_{(1, y)} \eta=\left\{\left(\xi-\mu, \mu_{\Omega}(y)\right) \mid \xi \in \mathbb{C}\left(\mathfrak{g}^{\mathbb{C}}\right)_{y}, \mu \in \mathbb{C} \mathfrak{g}\right\}$. We find $\xi \in \mathbb{C}\left(\mathfrak{g}^{\mathbb{C}}\right)_{y}, \mu \in \mathbb{C g}$ such that $W_{0}=\left(\xi-\mu, \mu_{\Omega}(y)+d_{y} \Phi_{0}\left(V_{0}\right)\right)$. But $W_{0} \in T_{(1, y)}^{1,0}\left(G^{\mathbb{C}} \times \Omega\right)$ and since $\mathfrak{g}$ is totally real in $\mathfrak{g}^{\mathbb{C}}$, we get $\mathbb{C} \mathfrak{g} \cap T_{1}^{1,0} G^{\mathbb{C}}=\{0\}$ and $\mu=0$. Because $\Phi_{0}$ is a CR embedding, this implies $V_{0} \in T_{y}^{1,0} \Omega$ and shows that $\Phi$ is a CR embedding.

Now let us consider the universality condition. We will assume $U=\Omega$, the general case is analogous. Let $Y$ be a complex manifold with holomorphic $G^{\mathbb{C}}$ action and $f: \Omega \rightarrow Y$ a $G$-equivariant CR map.

We define $F: \Omega^{\mathbb{C}} \rightarrow Y, h x \mapsto h f(x)$ for $h \in G^{\mathbb{C}}, x \in \Omega$. For this to be well-defined, we need to check that $h \in\left(G^{\mathbb{C}}\right)_{x}$ implies $h \in\left(G^{\mathbb{C}}\right)_{f(x)}$. 
Since $x \in \Omega$, we have $\left(G^{\mathbb{C}}\right)_{x}=\left(G_{x}\right)^{\mathbb{C}}$ and because the action of $G^{\mathbb{C}}$ on $Y$ is holomorphic, $G_{x} \subset\left(G^{\mathbb{C}}\right)_{f(x)}$ implies $\left(G^{\mathbb{C}}\right)_{x}=\left(G_{x}\right)^{\mathbb{C}} \subset\left(G^{\mathbb{C}}\right)_{f(x)}$.

We need to show that $F$ is holomorphic. Consider the commuting diagram

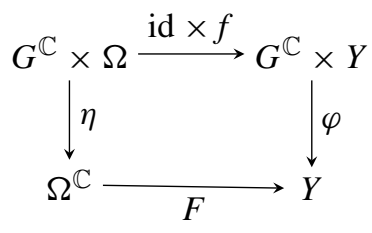

where $\varphi$ is the action map. Now $F$ is holomorphic if and only if $F \circ \eta$ is CR, which follows from the diagram.

We want to give a global version of this local statement. For this, we consider the union over all slice extensions and identify the overlapping parts. We formalize this as follows.

Around every $x \in X$, there exists a slice extension $\Omega_{x}^{\mathbb{C}}$. We may cover $X$ with countably many sets $\Omega_{i}$ with extensions $\Omega_{i}^{\mathbb{C}}$ for $i \in \mathbb{N}$.

For $i, j \in \mathbb{N}$ with $\Omega_{i} \cap \Omega_{j} \neq \emptyset$, define the open subset

$$
\Omega_{i j}^{\mathbb{C}}:=G^{\mathbb{C}} \cdot\left(\Omega_{i} \cap \Omega_{j}\right) \subset \Omega_{i}^{\mathbb{C}} .
$$

Then the identity map $\Omega_{i} \cap \Omega_{j} \rightarrow \Omega_{i} \cap \Omega_{j}$ extends to a unique $G^{\mathbb{C}}$-equivariant holomorphic map $\varphi_{j i}: \Omega_{i j}^{\mathbb{C}} \mapsto \Omega_{j i}^{\mathbb{C}}$, using Theorem 1.4.

Because of the uniqueness, we get $\varphi_{i i}=\mathrm{id}_{\Omega_{i}^{\mathbb{C}}}$ and $\varphi_{k j} \circ \varphi_{j i}=\varphi_{k i}$ on the open subset $G^{\mathbb{C}}\left(\Omega_{i} \cap \Omega_{j} \cap \Omega_{k}\right) \subset \Omega_{i}^{\mathbb{C}}$. This also implies $\varphi_{i j}=\varphi_{j i}^{-1}$.

Now define

$$
Z:=\bigcup_{i \in \mathbb{N}} \Omega_{i}^{\mathbb{C}} / \sim
$$

where $x \in \Omega_{i}^{\mathbb{C}}$ and $y \in \Omega_{j}^{\mathbb{C}}$ are equivalent if $\Omega_{i} \cap \Omega_{j} \neq \emptyset$ and $\varphi_{j i}(x)=y$. Because of the remarks above, this does indeed define an equivalence relation.

Define $Z_{0}:=\bigcup_{i \in \mathbb{N}} \Omega_{i}^{\mathbb{C}}$ to be the disjoint union over the $\Omega_{i}^{\mathbb{C}}$, the quotient map $\pi: Z_{0} \rightarrow Z$ and equip $Z$ with the quotient topology.

Lemma 1.5. The space $Z$ is Hausdorff and second countable.

Proof. Fix $i \in \mathbb{N}$ and let $U \subset \Omega_{i}^{\mathbb{C}}$ be open. Then $\pi^{-1}(\pi(U)) \cap \Omega_{j}^{\mathbb{C}}=\varphi_{j i}\left(U \cap \Omega_{i j}\right)$, which shows that $\pi^{-1}(\pi(U))$ is open.

We show that $Z$ is Hausdorff. If $U, V \subset \Omega_{i}^{\mathbb{C}}$ are disjoint, then $\pi^{-1}(\pi(U))$ and $\pi^{-1}(\pi(V))$ are also disjoint.

It therefore only remains to treat the case where $x \in \Omega_{1}^{\mathbb{C}} \backslash \Omega_{12}^{\mathbb{C}}$ and $y \in \Omega_{2}^{\mathbb{C}} \backslash \Omega_{21}^{\mathbb{C}}$.

First assume that $x \in \Omega_{1} \subset \Omega_{1}^{\mathbb{C}}$ and $y \in \Omega_{2} \subset \Omega_{2}^{\mathbb{C}}$. Then $x$ and $y$ can not be in the same $G$-orbit in $X$, since $\Omega_{1} \backslash \Omega_{12}^{\mathbb{C}}$ is a $G$-invariant neighborhood of $x$ not containing $y$. Using the existence of slices on $X$, we find open, $G$-invariant disjoint neighborhoods $U_{x}$ and $U_{y}$ of $x$ and $y$ in $X$ with $U_{x} \subset \Omega_{1}$ and $U_{y} \subset \Omega_{2}$. 
Define the open subsets $W_{x}:=G^{\mathbb{C}} \cdot U_{x}$ and $W_{y}:=G^{\mathbb{C}} \cdot U_{y}$ of $\Omega_{1}^{\mathbb{C}}$ and $\Omega_{2}^{\mathbb{C}}$. Assume that $\pi\left(W_{x}\right)$ and $\pi\left(W_{y}\right)$ are not disjoint. Then we find $x_{0} \in W_{x} \cap \Omega_{12}^{\mathbb{C}}$ and $y_{0} \in W_{y} \cap \Omega_{21}^{\mathbb{C}}$ such that $x_{0}$ and $y_{0}$ are equivalent. There exists $h \in G^{\mathbb{C}}$ such that $h x_{0} \in \Omega_{1} \cap W_{x}=U_{x}$ and since $\varphi_{21}\left(\Omega_{1}\right) \subset \Omega_{2}$, we conclude $h y_{0} \in \Omega_{2} \cap W_{y}=U_{y}$. But $h x_{0}$ and $h y_{0}$ are equivalent if and only if $h x_{0}=h y_{0}$ in $X$, implying that $U_{x}$ and $U_{y}$ are not disjoint, which is a contradiction.

The general case now follows because the sets $W_{h_{x} x}$ and $W_{h_{y} y}$ we constructed are $G^{\mathbb{C}}$-invariant and every $G^{\mathbb{C}}$-orbit in $\Omega_{i}^{\mathbb{C}}$ intersects $\Omega_{i}$.

Now $Z$ is second countable because the $\Omega_{i}^{\mathbb{C}}$ are second countable.

The $\Omega_{i}^{\mathbb{C}}$ give $Z$ the structure of a complex manifold. The $G^{\mathbb{C}}$-action on $Z$ is holomorphic because the $G^{\mathbb{C}}$-actions on the $\Omega_{i}^{\mathbb{C}}$ are holomorphic. The CR embeddings $\Phi_{i}: \Omega_{i} \rightarrow \Omega_{i}^{\mathbb{C}}$ extend to a CR embedding $\Phi: X \rightarrow Z$.

We summarize our results in the following Theorem.

Theorem 1.6. Let $G$ be a subgroup of its universal complexification and $X$ a $C R$ manifold with proper, transversal $C R G$-action. Then the map $\Phi: X \rightarrow Z$ is a $G$-equivariant $C R$ embedding of $X$ into the complex manifold $Z$.

Furthermore, for every $G$-invariant open subset $U \subset X$, the set $\left(G^{\mathbb{C}} \Phi(U), \Phi\right)$ is the universal equivariant extension of $U$. In particular, the manifold $(Z, \Phi)$ is the universal equivariant extension of $X$.

Proof. It remains to prove the universality condition. We will again assume $U=X$ and take a $G$-equivariant CR map $f: X \rightarrow Y$. For every $x \in X$, the map $f$ extends to a $G^{\mathbb{C}}$-equivariant holomorphic map $F_{i}: \Omega_{i}^{\mathbb{C}} \rightarrow Y$ and we define $F: Z \rightarrow Y$ via $F(y)=F_{i}(y)$ if $y \in \Omega_{i}^{\mathbb{C}}$. We need to show that this is well-defined. Consider $\Omega_{i}^{\mathbb{C}}$ and $\Omega_{j}^{\mathbb{C}}$ with $\Omega_{i} \cap \Omega_{j} \neq \emptyset$. But then $F_{i}: \Omega_{i j}^{\mathbb{C}} \rightarrow Y$ and $F_{j} \circ \varphi_{j i}: \Omega_{i j}^{\mathbb{C}} \rightarrow Y$ are both extensions of the same function $f$ on $\Omega_{i} \cap \Omega_{j}$, hence $F_{i}=F_{j} \circ \varphi_{j i}$ and $F$ is well-defined.

We now consider the quotient $X / G$, using that $X / G=Z / G^{\mathbb{C}}$, and show that it has the structure of a complex space. Since the action of $G^{\mathbb{C}}$ on $Z$ is generally not proper, we need to prove the existence of holomorphic slices, i.e. for every $z \in Z$, there exists a $\left(G^{\mathbb{C}}\right)_{z}$-invariant complex submanifold $S_{C}$ of $Z$ with $z \in S_{C}$ such that the map $G^{\mathbb{C}} \times{ }^{\left(G^{\mathbb{C}}\right)_{z}} S_{C} \rightarrow Z,[h, w] \mapsto h w$ is biholomorphic onto an open subset of $Z$.

Definition. Let $Z$ be a complex manifold with an action of a complex reductive group $K^{\mathbb{C}}$, where $K$ is a maximal compact subgroup of $K^{\mathbb{C}}$. An open subset $W \subset Z$ is called $K$-orbit convex if $W$ is $K$-invariant and for all $z \in W, \xi \in \mathfrak{k}$ the set $\{t \in \mathbb{R} \mid \exp (i t \xi) z \in W\}$ is connected.

If $Z, Y$ are complex manifolds with $K^{\mathbb{C}}$-action, $W \subset Z$ is $K$-orbit convex and $f: W \rightarrow Y$ is a $K$-equivariant holomorphic map, then we may define $F: K^{\mathbb{C}} W \rightarrow$ $Y$ by setting $F(k z):=k f(z)$ for $k \in K^{\mathbb{C}}$ and $z \in W$. The identity theorem for holomorphic functions then gives that $F$ is well-defined. 
Proposition 1.7. Let $G$ be a subgroup of its universal complexification $G^{\mathbb{C}}$ and $X$ a CR manifold with transversal, proper $G$-action. Let $x \in X, L=G_{x}$ and $\Omega^{\mathbb{C}}=G^{\mathbb{C}} \times^{L^{\mathbb{C}}} S$ a slice extension around $x$. Then there exists a holomorphic slice $S_{C}$ for the $G^{\mathbb{C}}$-action on $\Omega^{\mathbb{C}}$.

Proof. The group $G^{\mathbb{C}}$ is a Stein manifold [11, 1 Proposition] and $L^{\mathbb{C}}$ is complex reductive, hence $G^{\mathbb{C}} / L^{\mathbb{C}}$ is a Stein manifold [18]. We have an $L^{\mathbb{C}}$-action on $G^{\mathbb{C}} / L^{\mathbb{C}}$ via the multiplication from the left. Since $L^{\mathbb{C}}$ is complex reductive and $1 \cdot L^{\mathbb{C}} \in$ $G^{\mathbb{C}} / L^{\mathbb{C}}$ is a fixed point, we find an $L^{\mathbb{C}}$-invariant neighborhood $U$ of $1 \cdot L^{\mathbb{C}}$ and a $L^{\mathbb{C}}$-equivariant embedding of $U$ onto an open subset $U_{V}$ of an $L^{\mathbb{C}}$-representation $V$ [22, Theorem 5.2 and Remark 5.4]. Since $1 \cdot L^{\mathbb{C}}$ is a fixed point, translation by $1 \cdot L^{\mathbb{C}} \in U_{V}$ is $L^{\mathbb{C}}$-equivariant and we may assume that $1 \cdot L^{\mathbb{C}}$ gets mapped to $0 \in V$.

Now $L$ acts by unitary transformations on $V$ for a suitable inner product. Applying [10, 3.4 Proposition] to the inner product, we conclude that $0 \in V$ has a basis of $L$-orbit convex neighborhoods.

The inverse image of an $L$-orbit convex neighborhood of $1 \cdot L^{\mathbb{C}}$ via the $L^{\mathbb{C}}$ equivariant quotient map $G^{\mathbb{C}} /\left(L^{0}\right)^{\mathbb{C}} \rightarrow G^{\mathbb{C}} / L^{\mathbb{C}}$ is an $L$-orbit convex neighborhood $\tilde{W}$ of $1 \cdot\left(L^{0}\right)^{\mathbb{C}}$ in $G^{\mathbb{C}} /\left(L^{0}\right)^{\mathbb{C}}$ which is invariant under the $L^{\mathbb{C}}$-action from the right.

Now if $\tilde{W}$ is as above and $W_{0}$ is an $L^{\mathbb{C}}$-invariant neighborhood of $x \in S$, then the image of $\tilde{W} \times W_{0}$ is an $L$-orbit convex neighborhood of $x$ in $G^{\mathbb{C}} \times{ }^{L^{\mathbb{C}}} S$. We conclude that $x \in G^{\mathbb{C}} \times{ }^{L^{\mathbb{C}}} S$ has an $L$-orbit convex neighborhood basis.

Now take some $L$-orbit convex neighborhood $W_{1}$ of $x$ and an open $L$ invariant neighborhood $W_{2}$ of $0 \in T_{x} \Omega^{\mathbb{C}}$ with an $L$-equivariant biholomorphic map $\varphi: W_{1} \rightarrow W_{2}$. We may extend $\varphi$ to a $L^{\mathbb{C}}$-equivariant map $\Phi: L^{\mathbb{C}} W_{1} \rightarrow L^{\mathbb{C}} W_{2}$.

Apply [10, 3.4 Proposiiton] on the origin in $T_{x} \Omega^{\mathbb{C}}$ and find some $L$-orbit convex neighborhood $\tilde{W}_{2} \subset W_{2}$. Then extend $\varphi^{-1}$ to a $L^{\mathbb{C}}$-equivariant map $\tilde{\Phi}: L^{\mathbb{C}} \tilde{W}_{2} \rightarrow$ $L^{\mathbb{C}} W_{1}$. Set $U_{1}=L^{\mathbb{C}} \tilde{\Phi}(\tilde{W})$ and $U_{2}=\tilde{W}$, then $\tilde{\Phi}$ is inverse to $\Phi: U_{1} \rightarrow U_{2}$ because of equivariance.

By choosing an $L^{\mathbb{C}}$-invariant subspace of $T_{x} \Omega^{\mathbb{C}}$ which is perpendicular to $\mathfrak{l}^{\mathbb{C}}(x)$, we find an $L^{\mathbb{C}}$-invariant complex submanifold $S_{C}$ of $\Omega^{\mathbb{C}}$ through $x$ such that the induced map $\eta: G^{\mathbb{C}} \times{ }^{L^{\mathbb{C}}} S_{C} \rightarrow \Omega^{\mathbb{C}}$ is an immersion in [1, $\left.x\right]$.

We find some $L$-invariant neighborhood $\tilde{W}$ of $x$ in $\Omega^{\mathbb{C}}$ and an $L$-equivariant holomorphic map $\tilde{\eta}: \tilde{W} \rightarrow G^{\mathbb{C}} \times{ }^{L^{\mathbb{C}}} S_{C}$ with $\eta \circ \tilde{\eta}=$ Id.

Write $\Omega^{\mathbb{C}}=G^{\mathbb{C}} \times{ }^{L^{\mathbb{C}}} S$, then $\left.\tilde{\eta}\right|_{S \cap \tilde{W}}$ is an $L^{\mathbb{C}}$-equivariant map, which extends to a $G^{\mathbb{C}}$-equivariant map $\bar{\eta}$ on the open set $G^{\mathbb{C}} \cdot(\tilde{W} \cap S)$. Because of equivariance, we get $\eta \circ \bar{\eta}=\operatorname{Id}$ on $G^{\mathbb{C}} \cdot(\tilde{W} \cap S)$ and $\eta$ is biholomorphic after possibly shrinking $S_{C}$.

Theorem 1.8. Let $G$ be a closed subgroup of its universal complexification and $X$ a CR manifold with transversal, proper $C R G$-action. Then $X / G$ is a complex space, such that the sheaf of holomorphic functions on $X / G$ is given by the sheaf of $G$-invariant $C R$ functions on $X$. 
Proof. Let $x \in X$ and let $\Omega^{\mathbb{C}}$ be a slice extension. Proposition 1.7 then implies that locally, $\Omega / G=\Omega^{\mathbb{C}} / G^{\mathbb{C}}=S_{C} / L^{\mathbb{C}}$, where $S_{C}$ may be realized as an open subset of an $L^{\mathbb{C}}$-representation $V$. Now $L^{\mathbb{C}}$ acts as a finite group on $S_{C}$ and $V$, hence $V / L^{\mathbb{C}}$ is an affine variety, giving $X / G$ the structure of a complex space.

Because of the universality condition, every $G$-invariant CR map on $\Omega$ extends to a unique $G^{\mathbb{C}}$-invariant holomorphic function on $\Omega^{\mathbb{C}}$.

Remark. We have actually shown that $X / G$ is a complex orbifold.

Remark. If $X$ is a CR manifold with proper, transversal and locally free $G$-action, then the $G$-action on $G^{\mathbb{C}} \times X$ is free, proper and transversal, hence $G^{\mathbb{C}} \times{ }^{G} X$ is a complex manifold. Writing $X=G \times^{G} X$ and applying Lemma 1.2, one gets that $X \rightarrow G^{\mathbb{C}} \times^{G} X$ is an embedding. From the construction, it follows that $G^{\mathbb{C}} \times^{G} X$ is the universal equivariant extension of $X$.

\section{Pseudoconvexity}

In general, we need to impose additional conditions on a $\mathrm{CR}$ manifold to ensure the existence of an embedding into $\mathbb{C}^{n}$.

In the presence of a $G$-action, we will generalize the notion of pseudoconvexity to CR manifolds of higher codimension and establish a link between strongly pseudoconvex CR manifolds and positive line bundles.

Definition. Let $X$ be a CR manifold of dimension $(2 n, d)$ with an action of a Lie group $G$. We say that the action of $G$ on $X$ is transversal of codimension one if $\operatorname{dim} \mathfrak{g}(x)=d-1$ for all $x \in X$ and

$$
\mathfrak{g}(x) \cap\left(T_{x}^{1,0} X \oplus T_{x}^{0,1} X\right)=\{0\} .
$$

Example. Let $G$ be a Lie group and $M$ a CR manifold of dimension $(2 n, d)$ with transversal CR action of $G$. Let $X$ be a $G$-invariant hypersurface of $M$, then $X$ is a CR manifold of dimension $(2(n-1), d+1)$ with $G$-action which is transversal of codimension one.

To see this, define $W_{x}=\left(T_{x}^{1,0} M \oplus T_{x}^{0,1} M\right) \cap T_{x} M$, then

$$
\operatorname{dim} W_{x}+\operatorname{dim} T_{x} X=\operatorname{dim}\left(T_{x} X+W_{x}\right)+\operatorname{dim}\left(T_{x} X \cap W_{x}\right) .
$$

Since $X$ is $G$-invariant, $T_{x} X$ does contain $\mathfrak{g}(x)$ and $T_{x} X+W_{x}=T_{x} M$. This implies $\operatorname{dim}\left(T_{x} X \cap W_{x}\right)=2 n-1$.

Now every $W_{x}$ has a complex structure, which we denote by $J_{x}$. Define $V_{x}:=$ $T_{x} X \cap W_{x}$, then

$$
\operatorname{dim}\left(V_{x} \cap J_{x} V_{x}\right)=\operatorname{dim} V_{x}+\operatorname{dim} J_{x} V_{x}-\operatorname{dim}\left(V_{x}+J_{x} V_{x}\right),
$$

hence $\operatorname{dim}\left(V_{x} \cap J_{x} V_{x}\right)=2(n-1)$. 
Let $X$ be a CR manifold with locally free action of a Lie group $G$ which is transversal of codimension one and assume that $X$ is orientable.

Let $L$ be a line bundle transversal to $\mathfrak{g}(x) \oplus\left(T_{x}^{1,0} X \oplus T_{x}^{0,1} X\right) \cap T_{x} X$ in $T X$. Since $\left(T^{1,0} X \oplus T^{0,1} X\right) \cap T X$ is a complex vector space bundle over $X$, it is orientable. Since the action is locally free, the bundle $\bigcup_{x} \mathfrak{g}(x)$ is trivial and $L$ is orientable, hence trivial.

We conclude that in this case, we always find transversal vector fields, i.e. a vector field $T$ on $X$ such that $\mathbb{C} T(x) \oplus \mathbb{C} \mathfrak{g}(x) \oplus T_{x}^{1,0} X \oplus T_{x}^{0,1} X=\mathbb{C} T_{x} X$.

In fact, the above argument shows that, under the above conditions on the group action, $X$ is orientable if and only if there exist transversal vector fields.

From now on, let $X$ be a CR manifold with group action of $G$ which is transversal of codimension one and let $T$ be a transversal vector field on $X$. We define a 1-form on $X$ via $\alpha(T)=1$ and $\alpha\left(\mathfrak{g}(x) \oplus T_{x} X \cap\left(T^{1,0} X \oplus T^{0,1} X\right)\right)=0$, which we will call the projection onto $T$. For $V, W \in T^{1,0} X$, we define $\omega(V, W):=\frac{1}{2 i} d \alpha(V, \bar{W})$, by extending $d \alpha$ to a $\mathbb{C}$-bilinear map on every fiber of $\mathbb{C} T X$. By definition, $\omega$ is a hermitian form on $T^{1,0} X$.

Definition. Let $T$ be a transversal vector field on $X$, the form $\alpha$ the projection onto $T$ and $\omega$ be the induced hermitian form. We say that $T$ is strongly pseudoconvex if $\omega$ is positive definite. We call $X$ strongly CR-pseudoconvex if there exists a strongly pseudoconvex vector field $T$ on $X$.

Note that if $\mathrm{X}$ is of $\mathrm{CR}$ codimension one with trivial group action, this is the usual definition of strong pseudoconvexity.

For $V, W \in \mathcal{C}^{\infty}\left(X, T^{1,0} X\right)$, we get

$$
d \alpha(V, \bar{W})=V \alpha(\bar{W})-\bar{W} \alpha(V)-\alpha([V, \bar{W}])=-\alpha([V, \bar{W}]) .
$$

Let $S$ be another transversal vector field. We get that $\alpha(S)$ is point-wise nonvanishing, therefore $\beta:=\alpha(S)^{-1} \cdot \alpha$ is the projection onto $S$. Equation (1) then implies

$$
\frac{1}{2 i} d \beta(V, \bar{W})=\frac{1}{2 i} \alpha(S)^{-1} \cdot d \alpha(V, \bar{W})
$$

Definition. We say that a vector field $T$ on a CR manifold $X$ is a CR vector field if the flow of $T$ acts by CR automorphisms.

Note that in general, there do not need to exist transversal CR vector fields.

Let $X$ be a CR manifold with action of a compact Lie group $K$ which is transversal of codimension one. Let $T$ be a transversal vector field and $\alpha$ the projection onto $T$.

The $K$-action leaves the bundle $\mathbb{C} \mathfrak{k}(x) \oplus T_{x}^{1,0} X \oplus T_{x}^{0,1} X$ invariant, hence for $k \in K$, we get $\alpha\left(d_{x} k(T(x))\right) \neq 0$. We say that $K$ preserves the orientation of $T$ if $\alpha\left(d_{x} k(T(x))\right)>0$ for all $k \in K$ and $x \in X$. Note that if $K$ is connected, this is always the case. 
Lemma 2.1. Let $X$ be a $C R$ manifold with $K$-action which is transversal with codimension one. Let $T$ be a transversal $C R$ vector field such that $K$ preserves the orientation of $T$. Then there exists a transversal $C R$ vector field $\tilde{T}$ which is $K$-invariant. If $T$ is strongly pseudoconvex, then also $\tilde{T}$ is strongly pseudoconvex.

Proof. Let $\tilde{T}(x):=\int_{K} d k\left(T\left(k^{-1} x\right)\right) d k$ be the vector field averaged over $K$. If $\alpha$ is the projection onto $T$, then $\alpha(\tilde{T})=\int_{K} \alpha\left(d k T\left(k^{-1} x\right)\right) d k$. Because $K$ preserves the orientation of $T$, we conclude $\alpha(\tilde{T})>0$. In particular, we get that $\tilde{T}$ is transversal.

The flow of $\tilde{T}$ is given by $\Phi_{t}^{\tilde{T}}(x)=\int_{K} k\left(\Phi_{t}^{T}\left(k^{-1} x\right)\right) d k$, where $\Phi_{t}^{T}$ is the flow of $T$, which implies that $\tilde{T}$ is CR. Strong pseudoconvexity of $\tilde{T}$ follows from Eq. (2).

Theorem 2.2. Let $X$ be a compact $C R$ manifold with locally free $K$-action which is transversal of codimension one. Let $T$ be a strongly pseudoconvex $K$-invariant $C R$ vector field. Then there exists a strongly pseudoconvex $K$-invariant $C R$ vector field $\tilde{T}$ such that the flow of $\tilde{T}$ defines an $S^{1}$-action.

Proof. Let $\alpha$ be the projection onto $T$. We will define a riemannian metric on $X$. Since the $K$-action is locally free, the bundle $\mathfrak{k}(x)$ is trivial and we choose a riemannian metric $h$ for it.

A vector $V \in T_{X} X$ can be decomposed as $V=V_{T}+V_{\mathfrak{k}}+V_{\mathbb{C}}+V_{\overline{\mathbb{C}}}$, where $V_{\mathbb{C}} \in T_{x}^{1,0} X, V_{\overline{\mathbb{C}}} \in T_{x}^{0,1} X, V_{T} \in \mathbb{R} T(x)$ and $V_{\mathfrak{k}} \in \mathfrak{k}(x)$. Define a riemannian metric via

$$
g(V, U)=\alpha\left(V_{T}\right) \alpha\left(U_{T}\right)+h\left(V_{\mathfrak{k}}, U_{\mathfrak{k}}\right)+\frac{1}{2 i} d \alpha\left(U_{\mathbb{C}}, \bar{V}_{\mathbb{C}}\right)-\frac{1}{2 i} d \alpha\left(U_{\overline{\mathbb{C}}}, \bar{V}_{\overline{\mathbb{C}}}\right) .
$$

Since $\frac{1}{2 i} d \alpha\left(U_{\mathbb{C}}, \bar{V}_{\mathbb{C}}\right)$ is positive definite on $T^{1,0} X$, the form $\frac{1}{2 i} d \alpha\left(U_{\overline{\mathbb{C}}}, \overline{V_{\overline{\mathbb{C}}}}\right)=$ $-\frac{1}{2 i} d \alpha\left(\overline{V_{\overline{\mathbb{C}}}}, U_{\overline{\mathbb{C}}}\right)$ is negative definite on $T^{0,1} X$.

Denote by $\Phi_{t}^{T}$ the flow of $T$. Since $T$ is $K$-invariant and CR, the flow $\Phi_{t}^{T}$ commutes with the $K$-action and leaves the bundles $T^{1,0} X$ and $T^{0,1} X$ invariant. This implies that $\alpha$ is invariant under $\Phi_{t}^{T}$ and that the flow acts by isometries with respect to $g$.

The isometry group Iso $(X)$ of $(X, g)$ acts properly on $X$ and since $X$ is compact, we conclude that $\operatorname{Iso}(X)$ is compact. Then the subgroup $U$ of $\operatorname{Iso}(X)$ given by the CR isometries which commute with the $K$-action is compact, as well.

Now $T$ defines a subgroup of $U$, which is contained in a torus and the result follows because the vector fields defining $S^{1}$-actions are dense in this torus.

Now let $X$ be a compact CR manifold with transversal, locally free CR action of $S^{1} \times K$. Denote $H=S^{1} \times K$, then $Y:=H^{\mathbb{C}} \times{ }^{H} X$ is a complex manifold. Now consider the CR manifold $\left(\mathbb{C} \times K^{\mathbb{C}}\right) \times X$ with $S^{1} \times K$-action given by $((s, k),(z, h, x)) \mapsto\left(z s^{-1}, h k^{-1}, s k x\right)$. This action is transversal, therefore $Z:=\left(\mathbb{C} \times K^{\mathbb{C}}\right) \times{ }^{H} X:=\left(\left(\mathbb{C} \times K^{\mathbb{C}}\right) \times X\right) / H$ is a complex space. With the same argument, $Z / K^{\mathbb{C}}=\mathbb{C} \times{ }^{S^{1}}(X / K)$ is a complex space, as well. The natural map 
$Y \rightarrow Z$ is an open holomorphic injective immersion.

From Proposition 1.7, we conclude the existence of holomorphic slices on $H^{\mathbb{C}} \times{ }^{H} X$. The following theorem will be the main motivation for our further study.

Theorem 2.3. Let $X$ be a compact $C R$ manifold with transversal, locally free, $C R$ $S^{1} \times K$-action such that the vector field $T$ induced by the $S^{1}$-action is strongly pseudoconvex. Then the set

$$
W:=\left\{[z, x] \in \mathbb{C} \times S^{1} X / K=Z /\left.K^{\mathbb{C}}|| z\right|^{2}<1\right\}
$$

is strongly pseudoconvex in $Z / K^{\mathbb{C}}$.

Proof. For the map $h: Z / K^{\mathbb{C}} \rightarrow \mathbb{R},[z, x] \mapsto|z|^{2}$, we have $W=\{p \in$ $\left.Z / K^{\mathbb{C}} \mid h(p)<1\right\}$.

Let $U$ be the preimage of $W$ under $Z \rightarrow Z / K^{\mathbb{C}}$. We pull back $h$ to a function on $Z$, which we will still denote by $h$. We have $U=\{z \in Z \mid h(z)<1\}$. The map $x \mapsto[1,1, x]$ is a $\mathrm{CR}$ embedding of $X$ and $T$ extends to a $\mathbb{C}^{*} \times K^{\mathbb{C}}$-invariant vector field on $Y$, which is the vector field induced by the $S^{1}$-action on $Y$.

The boundary $\partial U=\left\{[1, g, x] \in\left(\mathbb{C}^{*} \times K^{\mathbb{C}}\right) \times{ }^{S^{1} \times K} X\right\}=K^{\mathbb{C}} X$ is a hypersurface and therefore a CR submanifold of $Y$ with transversal $S^{1}$-action. We therefore have $\mathbb{C} T_{x} \partial U=T_{x}^{1,0} \partial U \oplus T_{x}^{0,1} \partial U \oplus \mathbb{C} T(x)$ and define the projection onto $T$ via $\alpha(T(x))=1$ and $\alpha\left(T^{1,0} \partial U \oplus T^{0,1} \partial U\right)=0$.

Take $y_{0} \in \partial W$ and let $\pi: Z \rightarrow Z / K^{\mathbb{C}}$ be the quotient map. We then find an $x_{0} \in X$ such that $\pi\left(x_{0}\right)=y_{0}$. Let $S$ be a holomorphic slice through $x_{0}$ for the $H^{\mathbb{C}}$-action and $\Omega=H^{\mathbb{C}} \times^{L} S$, where $L$ is the finite isotropy of $H^{\mathbb{C}}$ in $x_{0}$. We may assume that $S$ is biholomorphic to a ball and $\mathbb{C} T_{x_{0}} S=T_{x_{0}}^{1,0} X \oplus T_{x_{0}}^{0,1} X$, since actions of compact groups at fixed points can be linearised.

Let $[w, g, y]$ be the coordinates of $\left(\mathbb{C}^{*} \times K^{\mathbb{C}}\right) \times{ }^{L} S$ and take some smooth function $\varphi$ on $\Omega$ such that $h[w, g, y] \cdot e^{\varphi[w, g, y]}=|w|^{2}$. By construction, $\varphi$ is $H^{\mathbb{C}}$ invariant and $\Omega \cap \partial U$ is given by $\Phi[w, g, y]:=|w|^{2}-e^{\varphi[w, g, y]}=0$.

Let $\frac{\partial}{\partial w}$ be the complex vector field induced be the $\mathbb{C}^{*}$-component in $\Omega$, let $\left(\frac{\partial}{\partial z_{j}}\right)_{j=1, \ldots, m}$ be a basis for $T^{1,0} S$ and extend this to a basis $\left(\frac{\partial}{\partial w}, \frac{\partial}{\partial z_{j}}\right)_{j=1, \ldots, n}$ for $T^{1,0} \Omega$. This is always possible after shrinking $\Omega$.

We want to compute the CR structure of $\partial U \cap \Omega$ in terms of the above basis and are therefore considering the equation

$$
0=d \Phi\left(\sum_{j} a_{j} \frac{\partial}{\partial z_{j}}+b \frac{\partial}{\partial w}\right)=-\sum_{j} a_{j} e^{\varphi} \frac{\partial}{\partial z_{j}} \varphi+b \bar{w} .
$$

This implies that $Z_{j}=\frac{\partial}{\partial z_{j}}+\frac{w}{|w|^{2}} e^{\varphi} \frac{\partial}{\partial z_{j}} \varphi \frac{\partial}{\partial w}$ gives a basis for the CR structure. 
Rewriting $\frac{\partial}{\partial w}$ in Polar coordinates $(r, \theta)$ gives

$$
\frac{\partial}{\partial w}=\frac{1}{2} \frac{\bar{w}}{|w|} \frac{\partial}{\partial r}-\frac{1}{2} i \frac{\bar{w}}{|w|^{2}} \frac{\partial}{\partial \theta} .
$$

We get $Z_{j}=\frac{\partial}{\partial z_{j}}+\frac{1}{2|w|} \frac{\partial}{\partial z_{j}} \varphi \frac{\partial}{\partial r}-\frac{1}{2} i \frac{\partial}{\partial z_{j}} \varphi \frac{\partial}{\partial \theta}$, using that $\frac{e^{\varphi}}{|w|^{2}}=1$ on the boundary of $U$. On $\partial U$, the projection $\alpha$ is given by $\alpha\left(\frac{\partial}{\partial \theta}\right)=\alpha(T)=1$ and $\alpha\left(Z_{j}\right)=\alpha\left(\bar{Z}_{j}\right)=$ 0 , therefore

$$
\alpha=d \theta+\sum_{j} \frac{1}{2} i \frac{\partial}{\partial z_{j}} \varphi d z_{j}-\sum_{j} \frac{1}{2} i \frac{\partial}{\partial \bar{z}_{j}} \varphi d \bar{z}_{j} .
$$

Now $\mathbb{C} T_{x_{0}} S=T_{x_{0}}^{1,0} X \oplus T_{x_{0}}^{0,1} X$, hence for $v, w \in T_{x_{0}}^{1,0} X$, we have the equation

$$
\frac{1}{2 i} d \alpha(v, \bar{w})=-\sum_{i j} \frac{1}{2} \frac{\partial^{2}}{\partial z_{j} \partial \bar{z}_{i}} \varphi(v, \bar{w}),
$$

which implies that $-\varphi$ is strictly plurisubharmonic in a neighborhood of $x_{0}$ in $S$. After shrinking $S$, we may assume that $-\varphi$ is strictly plurisubharmonic on $S$.

We restrict $\varphi$ to an $L$-invariant function on $S$, write $\Omega / K^{\mathbb{C}}=\mathbb{C}^{*} \times{ }^{L} S$ and

$$
W \cap\left(\Omega / K^{\mathbb{C}}\right)=\left\{[w, y] \in \mathbb{C}^{*} \times\left.^{L} S|| w\right|^{2}<e^{\varphi(y)}\right\} .
$$

Define the map $\pi_{L}: \mathbb{C}^{*} \times S \rightarrow \mathbb{C}^{*} \times{ }^{L} S$, then the set $\pi_{L}^{-1}(W)=$ $\left\{\left.(w, y)|| w\right|^{2}<e^{\tilde{\varphi}(y)}\right\}$ is a Hartogs domain, which is strongly pseudoconvex because $-\varphi$ is strictly plurisubharmonic and $S$ is biholomorphic to a ball. Now the boundary of $\pi_{L}^{-1}(W)$ in $\mathbb{C}^{*} \times S$ is given by a strongly plurisubharmonic $L$ invariant map $\rho$. Then $\rho$ defines a map $\bar{\rho}$ on $\mathbb{C}^{*} \times{ }^{L} S$, which is plurisubharmonic $[8$, Satz 3$]$ and it only remains to prove that it remains plurisubharmonic under perturbations. But this follows using [8, Satz 3] again and the fact that $\pi_{L}$ is proper.

Remark. We have actually shown that the orbifold line bundle $Z / K^{\mathbb{C}} \rightarrow Y /\left(\mathbb{C}^{*} \times\right.$ $K^{\mathbb{C}}$ ) is weakly negative (see Sect. 4).

\section{Equivariant embeddings}

In this section we prove an embedding theorem for strongly pseudoconvex CR manifolds.

Let $H$ be a subgroup of its universal complexification $H^{\mathbb{C}}$ and assume that $H^{\mathbb{C}}$ is complex reductive. Let $G$ be a normal subgroup of $H$ such that $H=G \rtimes S^{1}$. This is a slightly more general setting then the direct product $K \times S^{1}$ we considered before. The group $H^{\mathbb{C}}$ is isomorphic to $G^{\mathbb{C}} \rtimes \mathbb{C}^{*}$ and $\mathbb{C}^{*}$ is a closed subgroup of $H^{\mathbb{C}}$.

For $h \in H$, we denote by $h_{S} \in H / G \cong S^{1}$ the image of $h$ in the quotient and for $h \in H^{\mathbb{C}}$ we write $h_{C} \in H^{\mathbb{C}} / G^{\mathbb{C}} \cong \mathbb{C}^{*}$, respectively. Note that the maps $h \mapsto h_{S}$ and $h \mapsto h_{C}$ are group morphisms. 
Now let $X$ be a CR manifold with transversal, proper CR action of $H$ and assume that $H_{x}^{0}<G_{x}$ in every point $x \in X$. Denote by $T$ the vector field induced by the $S^{1}$-action. The condition on the isotropy groups is equivalent to $\mathbb{C} T(x) \oplus$ $\mathbb{C} \mathfrak{g}(x) \oplus T_{x}^{1,0} X \oplus T_{x}^{0,1} X=\mathbb{C} T_{x} X$.

Note that the condition $H_{x}^{0}<G_{x}^{0}$ is always satisfied if the $H$-action is locally free.

We have an $H$-action on the $\mathrm{CR}$ manifold $\mathbb{C} \times X$ given by $(h,(z, x)) \mapsto$ $\left(z h_{S}^{-1}, h x\right)$. This action is proper and $\mathrm{CR}$ and $H_{x}^{0}<G_{x}^{0}$ implies that it is transversal. We conclude that

$$
Z:=(\mathbb{C} \times X) / H \cong \mathbb{C} \times S^{1}(X / G)
$$

is a complex space.

We may embed $X$ into its universal equivariant extension $Y$ as in Theorem 1.6. We define the complex space $(\mathbb{C} \times Y) / H^{\mathbb{C}}=\mathbb{C} \times \mathbb{C}^{*}\left(Y / G^{\mathbb{C}}\right)$ in the same manner as above and show that the natural map $(\mathbb{C} \times X) / H \rightarrow(\mathbb{C} \times Y) / H^{\mathbb{C}}$ is an isomorphism.

It is sufficient to consider a slice neighborhood $\Omega=H \times{ }^{H_{x}} S$ of $X$ and show that the map $\varphi:(\mathbb{C} \times \Omega) / H \rightarrow\left(\mathbb{C} \times \Omega^{\mathbb{C}}\right) / H^{\mathbb{C}}$ is biholomorphic. But the inverse of $\varphi$ is given by $\varphi^{-1}:\left(\mathbb{C} \times\left(H^{\mathbb{C}} \times H_{x}^{\mathbb{C}} S\right)\right) / H^{\mathbb{C}} \rightarrow\left(\mathbb{C} \times\left(H \times H_{x} S\right)\right) / H$ with $[z,[h, s]] \mapsto\left[z h_{C},[1, s]\right]$.

Definition. We say that $X$ is strongly pseudoconvex if the set

$$
Z_{\Delta}:=\left\{[w, z] \in \mathbb{C} \times S^{1} X / G|| w \mid<1\right\}
$$

is strongly pseudoconvex in $Z$.

The following construction, including Corollary 3.2, is based on ideas of Grauert, see [7, 3.2]. We will reformulate it here, in our context.

Definition. Let $M$ be a set with $S^{1}$-action, $U \subset M$ a subset and $V$ a vector space. We say that a map $f: U \rightarrow V$ is of order $d \in \mathbb{Z}$ if for all $s \in S^{1}$ and $m \in U$ such that $s m \in U$, we have $f(s m)=s^{d} f(m)$.

Consider the complex space $X / H=Y / H^{\mathbb{C}}$. The structure sheaf $\mathcal{O}_{Y}^{H}$ of $Y$ is given by the sheaf of $H$-invariant holomorphic functions on $Y$. Given a coherent analytic sheaf $\mathcal{G}^{Y}$ over $Y / H^{\mathbb{C}}$, we define a sheaf $\mathcal{G}^{Z}$ over $Z$ as follows. The holomorphic map $p: Z \rightarrow Y / H^{\mathbb{C}}$ gives the structure sheaf $\mathcal{O}_{Z}$ of $Z$ the structure of an $\mathcal{O}_{Y}^{H}$-module. Define

$$
\mathcal{G}^{Z}(U)=\mathcal{G}^{Y}(p(U)) \otimes_{\mathcal{O}_{Y}^{H}(p(U))} \mathcal{O}_{Z}(U) .
$$

Now $\mathcal{G}^{Z}$ is coherent and analytic on $Z$ because $\mathcal{G}^{Y}$ is coherent and analytic on $Y / H^{\mathbb{C}}$.

The map $Y / H^{\mathbb{C}} \rightarrow Z, y \mapsto[0, y]$ is an embedding of $Y / H^{\mathbb{C}}$ as the analytic set of $\mathbb{C}^{*}$-fixed points. Define the coherent sheaf $\mathcal{O}_{Z}^{Y}$ of holomorphic functions on 
$Z$ which vanish on $Y / H^{\mathbb{C}}$. We have the (non-analytic) subsheaf $\mathcal{O}_{Z, d}^{Y}$ of functions vanishing on $Y / H^{\mathbb{C}}$ which are of order $d$. The sheaves $\mathcal{O}_{Z}^{Y}$ and $\mathcal{O}_{Z, d}^{Y}$ then define analytic sheaves $\mathcal{O}_{Y}^{Y}(U)$ and $\mathcal{O}_{Y, d}^{Y}(U)$ on $Y / H^{\mathbb{C}}$ via pulling back with $p$.

Let $\mathcal{G}^{Y}$ be a coherent analytic sheaf over $Y / H^{\mathbb{C}}$. The natural map $\mathcal{O}_{Y, d}^{Y}(U) \rightarrow$ $\mathcal{O}_{Z}\left(p^{-1}(U)\right)$ induces the map

$$
A_{\leq d_{0}}: \bigoplus_{0 \leq d \leq d_{0}} H^{q}\left(Y / H^{\mathbb{C}}, \mathcal{G}^{Y} \otimes \mathcal{O}_{Y, d}^{Y}\right) \rightarrow H^{q}\left(Z, \mathcal{G}^{Z}\right)
$$

where $H^{q}$ denotes the $q$-th Čech cohomology.

We now define a map

$$
B_{d}: H^{q}\left(Z, \mathcal{G}_{Z}\right) \rightarrow H^{q}\left(Y / H^{\mathbb{C}}, \mathcal{G}^{Y} \otimes \mathcal{O}_{Y, d}^{Y}\right)
$$

as follows.

Let $U \subset Z$ be open with $U \cap Y / H^{\mathbb{C}} \neq \varnothing$ and $g: U \rightarrow \mathbb{C}$ a holomorphic function.

Let $\pi: \mathbb{C} \times Y \rightarrow(\mathbb{C} \times Y) / H^{\mathbb{C}}=Z$ be the quotient map, then $\bar{g}:=g \circ \pi$ is a $H^{\mathbb{C}}$-invariant holomorphic function on $\pi^{-1}(U)$. Consider the set $U_{0}=\{(0, w) \in$ $\left.\pi^{-1}(U)\right\}$, take $\left(0, w_{0}\right) \in U_{0}$ and develop $\bar{g}$ into a power series $\bar{g}=\sum_{d} z^{d} f_{d, w_{0}}(w)$ in some connected neighborhood of $\left(0, w_{0}\right)$ of the form $B_{w_{0}} \times \Omega_{w_{0}}$.

If $\left(0, w_{1}\right) \in U_{0}$ is another point such that $B_{w_{1}} \times \Omega_{w_{1}} \cap B_{w_{0}} \times \Omega_{w_{0}} \neq \emptyset$, we have $f_{d, w_{0}}=f_{d, w_{1}}$ on the intersection. We may therefore assume that $f_{d}$ is defined on $\Omega:=\bigcup_{(0, w) \in U_{0}} B_{w} \times \Omega_{w}$ and set $\bar{g}_{d}(z, w):=z^{d} f_{d}(w)$ on $\Omega$.

Now the $\bar{g}_{d}$ are actually $H^{\mathbb{C}}$-invariant, which we will see as follows. Set $\tilde{g}_{d}(h x):=\bar{g}_{d}(x)$ for $h \in H^{\mathbb{C}}, x \in \Omega$, we need to show that this is well-defined. For this, fix some $h \in H^{\mathbb{C}}$ and observe that every connected component of $h \Omega$ intersects $U_{0}$. For $\left(0, w_{0}\right) \in U_{0}$, we consider the power series expansions of $\bar{g}$ and $\bar{g} \circ h^{-1}$, which have to coincide because $\bar{g}$ is $H^{\mathbb{C}}$-invariant. For $c \in \mathbb{C}^{*}$, we compute

$$
\bar{g}_{d}\left(h^{-1}(c z, w)\right)=\bar{g}_{d}\left(c z h_{C}, h^{-1} w\right)=c^{d} \bar{g}_{d}\left(z h_{C}, h^{-1} w\right) .
$$

Comparing coefficients in the power series expansions then gives $\bar{g}_{d}=\bar{g}_{d} \circ h^{-1}$ on $\Omega \cap h \Omega$ and $\tilde{g}_{d}$ is well-defined.

We may therefore assume that the sets $B_{w} \times \Omega_{w}$ are $H^{\mathbb{C}}$-invariant. Since the $\bar{g}_{d}$ are of order $d$, they may be extended to functions on $\bigcup_{(0, w) \in U_{0}} \mathbb{C} \times \Omega_{w}$. Now the $\bar{g}_{d}$ define holomorphic functions $g_{d}$ on $p^{-1}\left(p\left(U \cap Y / H^{\mathbb{C}}\right)\right)$ of degree $d$.

For every open subset $U \subset Z$ with $U \cap Y / H^{\mathbb{C}} \neq \emptyset$, we have a map

$$
B_{d, U}: \mathcal{G}^{Z}(U) \rightarrow \mathcal{G}^{Y}\left(p\left(U \cap Y / H^{\mathbb{C}}\right)\right) \otimes \mathcal{O}_{Y, d}^{Y}\left(p\left(U \cap Y / H^{\mathbb{C}}\right)\right)
$$

by restriction in the first component and the construction from above in the second component.

Now we want to define $B_{d}$ on the cohomology groups. 
If $V$ is an open subset of $U$ with $V \cap Y / H^{\mathbb{C}} \neq \varnothing$, then $V \cap Y / H^{\mathbb{C}}$ is a subset of $U \cap Y / H^{\mathbb{C}}$ and from the above construction, we see

$$
B_{d, V}\left(\operatorname{res}_{V}^{U}(g)\right)=\operatorname{res}_{p\left(V \cap Y / H^{\mathbb{C}}\right)}^{p\left(U \cap Y / H^{\mathbb{C}}\right)} B_{d, U}(g) .
$$

Given a covering $\mathcal{V}$ of $Z$, we define a covering $\mathcal{V}^{Y}$ of $Y / H^{\mathbb{C}}$ via $V_{i}^{Y}:=p\left(V_{i} \cap\right.$ $\left.Y / H^{\mathbb{C}}\right)$,

if $V_{i} \cap Y / H^{\mathbb{C}} \neq \emptyset$. For a cochain $g \in C^{q}\left(\mathcal{V}, \mathcal{G}^{Z}\right)$ and a $q$-simplex $\sigma^{Y}$ for $\mathcal{V}^{Y}$, with corresponding simplex $\sigma$ for $\mathcal{V}$, we define $B_{d}(g)\left(\sigma^{Y}\right):=B_{d,|\sigma|}(g(\sigma))$.

Now if $G \in C^{q-1}\left(\mathcal{V}, \mathcal{G}^{Z}\right)$ is a cochain, $\sigma$ is a $q$-simplex for $\mathcal{V}$ and $\partial_{j} \sigma$ is the corresponding $(q-1)$-simplex by omitting the $j$-th set, then we have

$$
\begin{aligned}
B_{d,|\sigma|}(\delta G(\sigma)) & =B_{d,|\sigma|}\left(\sum_{j}(-1)^{j} \operatorname{res}_{|\sigma|}^{\left|\partial_{j} \sigma\right|}\left(G\left(\partial_{j} \sigma\right)\right)\right) \\
& =\sum_{j}(-1)^{j} \operatorname{res}_{p\left(|\sigma| \cap Y / H^{\mathbb{C}}\right)}^{p\left(\left|\partial_{j} \sigma\right| \cap Y / H^{\mathbb{C}}\right)}\left(B_{d,\left|\partial_{j} \sigma\right|}\left(G\left(\partial_{j} \sigma\right)\right)\right) .
\end{aligned}
$$

From this computation and Eq. (3), we conclude that the maps

$$
B_{d}: H^{q}\left(Z, \mathcal{G}^{Z}\right) \rightarrow H^{q}\left(Y / H^{\mathbb{C}}, \mathcal{G}^{Y} \otimes \mathcal{O}_{Y, d}^{Y}\right),
$$

and

$$
B_{\leq d_{0}}: H^{q}\left(Z, \mathcal{G}^{Z}\right) \rightarrow \bigoplus_{d \leq d_{0}} H^{q}\left(Y / H^{\mathbb{C}}, \mathcal{G}^{Y} \otimes \mathcal{O}_{Y, d}^{Y}\right)
$$

are well-defined, since they map cocycles to cocycles and coboundaries to coboundaries.

Since $B_{\leq d_{0}} \circ A_{\leq d_{0}}=\mathrm{Id}$, we conclude that $A_{\leq d_{0}}$ is injective for every $d_{0}$.

The following Proposition was proved by Grauert [6, Proposition 4] for the structure sheaf on complex manifolds and the proof also holds for this case using [5, Theorem 5.4], also see [7, Hilfssatz 1].

Proposition 3.1. Let $Z$ be a complex space and $\Omega \subset Z$ a strongly pseudoconvex, relatively compact set. Let $\mathcal{G}$ be a coherent analytic sheaf on $Z$. Then the complex vector spaces $H^{q}(\Omega, \mathcal{G})$ are finite-dimensional for $q>0$.

From Proposition 3.1 and the injectivity of $A_{\leq d_{0}}$, we conclude the following result.

Corollary 3.2. Let $X / G$ be compact, $X$ be strongly pseudoconvex and $\mathcal{G}^{Y}$ a coherent analytic sheaf on $Y / H^{\mathbb{C}}$. Then for every $q>0$, there exists a $d_{0}$ such that

$$
H^{q}\left(Y / H^{\mathbb{C}}, \mathcal{G}^{Y} \otimes \mathcal{O}_{Y, d}^{Y}\right)=0
$$

for all $d \geq d_{0}$. 
Let $V$ be a $H^{\mathbb{C}}$-representation. We may define a new $H^{\mathbb{C}}$-representation structure on $V$ via $(h, v) \mapsto h_{C}^{d} h v$, which we denote by $V_{d}$.

Note that for an open subset $U$ of $Y$, the $G^{\mathbb{C}}$-invariant holomorphic functions of order $d>0$ on $U$ are one-to-one with the holomorphic functions of order $d$ on $\mathbb{C} \times \mathbb{C}^{*} U / G^{\mathbb{C}}$.

Now let $\pi: Y \rightarrow Y / H^{\mathbb{C}}$ be the projection and $V$ a $H^{\mathbb{C}}$-representation. We define the sheaf $\mathcal{F}^{Y}$ on $Y / H^{\mathbb{C}}$ as

$$
\mathcal{F}^{Y}(U):=\left\{f: \pi^{-1}(U) \rightarrow V \mid f \text { holomorphic, } H^{\mathbb{C}} \text {-equivariant }\right\} .
$$

For $z \in Y / H^{\mathbb{C}}$ we set

$$
\mathcal{F}_{z, z}^{Y}(U):=\left\{f \in \mathcal{F}^{Y}(U) \mid f(z)=0 \text { and } d f(z)=0\right\}
$$

and for $z, w \in Y / H^{\mathbb{C}}$, consider

$$
\mathcal{F}_{z, w}^{Y}(U):=\left\{f \in \mathcal{F}^{Y}(U) \mid f(z)=f(w)=0\right\} .
$$

The existence of holomorphic slices (Proposition 1.7) also implies that every $H^{\mathbb{C}}$ orbit is analytic. This shows that for a fixed $y \in Y$, the sheaf $\mathcal{O}_{H^{\mathbb{C}} y}^{V}$ of holomorphic maps to $V$ which vanish on $H^{\mathbb{C}} y$ is a coherent analytic $H^{\mathbb{C}}$-sheaf (see [21]).

Given a point $x \in Y$, we find a slice $S_{C}$ which is a Stein manifold. Since $H^{\mathbb{C}}$ is complex reductive, we have that $H^{\mathbb{C}} \times{ }^{H_{y}^{\mathbb{C}}} S_{C}$ is Stein. Hence $Y / H^{\mathbb{C}}$ has a neighborhood basis such that the preimages under the quotient map are Stein. We may use [21, Theorem 3.1] to see that both sheaves above are coherent analytic sheaves on $Y / H^{\mathbb{C}}$.

Let $x \in X$ and $L=H_{x}$ with $L^{0} \subset G$. Then $L^{\mathbb{C}}$ acts from the right on $H^{\mathbb{C}} / G^{\mathbb{C}}$ as a finite group $N$. We call the order $|N|$ of the finite group $N$ the order of $\mathbb{C}^{*}$ in $x$. The map $h_{C} \mapsto h_{C}^{|N|}$ is an $N$-invariant map on $H^{\mathbb{C}} / G^{\mathbb{C}}$.

Proposition 3.3. Let $y \in X, L=H_{y}$ and $H^{\mathbb{C}} \times{ }^{L^{\mathbb{C}}} S_{C}$ be a holomorphic slice in $Y$. Let $l$ be the order of $\mathbb{C}^{*}$ in $x$. Then there exists a $H^{\mathbb{C}}$-representation $V$ and $a$ $H^{\mathbb{C}}$-equivariant holomorphic injective immersion $\varphi: H^{\mathbb{C}} \times{ }^{L^{\mathbb{C}}} S_{C} \rightarrow V$ such that, for every $m \in \mathbb{N}$, the map

$$
\begin{array}{r}
\Phi: H^{\mathbb{C}} \times{ }^{L^{\mathbb{C}}} S_{C} \rightarrow V_{m l} \times V_{(m+1) l} \\
{[h, z] \mapsto\left(h_{C}^{m l} \varphi[h, z], h_{C}^{(m+1) l} \varphi[h, z]\right)}
\end{array}
$$

is a well-defined, injective equivariant immersion on some open, $H^{\mathbb{C}}$-invariant neighborhood $U_{m}$ of $y$ in $Y$.

For all $y_{0} \in U_{m}$, the restricted map $\Phi: G^{\mathbb{C}} y_{0} \rightarrow V_{m l} \times V_{(m+1) l}$ is an embedding. 
Proof. We May assume that $S_{C} \subset T_{y} Y$ and $H^{\mathbb{C}} \times{ }^{L^{\mathbb{C}}} S_{C} \subset H^{\mathbb{C}} \times{ }^{L^{\mathbb{C}}} T_{y} Y$. The quotient $H^{\mathbb{C}} \times{ }^{L^{\mathbb{C}}} T_{y} Y$ is an affine variety with an action of the complex reductive group $H^{\mathbb{C}}$, therefore we find an equivariant holomorphic embedding into some representation $V$.

Let $\varphi: H^{\mathbb{C}} \times{ }^{L^{\mathbb{C}}} S_{C} \rightarrow V$ be the restriction of that embedding. The $L^{\mathbb{C}}$-orbits in $S_{C}$ are all finite. We conclude that all $H^{\mathbb{C}}$-orbits in $H^{\mathbb{C}} \times^{L^{\mathbb{C}}} S_{C}$ are also closed in $H^{\mathbb{C}} \times{ }^{L^{\mathbb{C}}} T_{y} Y$, hence $\varphi$ is an embedding on every $H^{\mathbb{C}}$-orbit.

Let $\Phi$ be defined as in the statement of the Proposition. Using that $\varphi$ is an equivariant immersion and therefore non-vanishing, a direct computation shows that $\Phi$ is injective.

Now consider $\tilde{z}:=[1, z]$, take $\xi \in \mathbb{C}=T_{1} \mathbb{C}^{*}, v \in \mathcal{G}^{\mathbb{C}}$ and $v \in T_{\tilde{z}} S_{C}$. Define $: w=\xi(\tilde{z})+v(\tilde{z})+v$, then

$$
d_{\tilde{z}} \Phi(w)=\left(l m \cdot \xi \cdot \varphi(\tilde{z})+d_{\tilde{z}} \varphi(w), l(m+1) \cdot \xi \cdot \varphi(\tilde{z})+d_{\tilde{z}} \varphi(w)\right)
$$

and because $\varphi(\tilde{z}) \neq 0$ and $\varphi$ is an immersion, one sees that $\Phi$ is an immersion in $\tilde{z}$. Because $\Phi$ is equivariant, it is an immersion in some invariant neighborhood $U_{m}$ of $H^{\mathbb{C}} y$.

For fixed $y_{0} \in U_{m}$, we get $\left.\Phi\right|_{G^{\mathbb{C}} y_{0}}=\left(c_{1} \varphi, c_{2} \varphi\right)$ for some constants $c_{1}$ and $c_{2}$, which is an embedding.

For fixed $y \in X$ with order $l$ of $\mathbb{C}^{*}$ in $y$, define $f_{m}: H^{\mathbb{C}} \times^{L^{\mathbb{C}}} S_{C} \rightarrow \mathbb{C}$, $[h, x] \mapsto h_{C}^{m l}$. Let $\varphi$ be the map as in Proposition 3.3, then $\varphi \otimes f_{m}$ defines an element in $\mathcal{F}^{Y} \otimes \mathcal{O}_{Y, m l}^{Y}$ (see (4)). Applying Corollary 3.2 to the sheaf (5) and choosing $m$ large enough, we find a $H^{\mathbb{C}}$-representation $V_{y}$, and a $H^{\mathbb{C}}$-equivariant holomorphic map $\Phi_{y}: Y \rightarrow V_{y}$ such that $\Phi_{y}=\left(f_{m} \varphi, f_{m+1} \varphi\right)$ on $H^{\mathbb{C}} y$ up to order 2. We therefore find a neighborhood $U_{y}$ of $y$, such that the map $\Phi_{y}: U_{y} \rightarrow V_{y}$ is an immersion on $U_{y}$, injective on $H^{\mathbb{C}} y$ and $\Phi_{y}: G^{\mathbb{C}} y \rightarrow V_{y}$ is an embedding.

Using the same argument for the trivial $H^{\mathbb{C}}$-representation $\mathbb{C}$ and $\varphi$ being a constant map, we may construct a $G^{\mathbb{C}}$-invariant holomorphic map $F: Y \rightarrow \mathbb{C}^{m}$ which does not vanish on $Y$ and every component $F_{i}$ of $F$ is of order $d_{i}>0$. Because $X / G$ is compact, we obtain $\inf _{x \in X}\|F(x)\|^{2}=c>0$.

Lemma 3.4. Let $\Phi_{y}, F$ be as above. Then there exist open, $H^{\mathbb{C}}$-invariant neighborhoods $U_{y}$ of $y$ in $Y$ and $W_{y}$ of $\left(F \times \Phi_{y}\right)(y)$ in $\mathbb{C}^{m} \backslash\{0\} \times V_{y}$ such that $\left(F \times \Phi_{y}\right): U_{y} \rightarrow W_{y}$ is an embedding. Furthermore, the set $W_{y}$ is saturated with respect to the quotient map $\mathbb{C}^{m} \backslash\{0\} \times V_{y} \rightarrow\left(\mathbb{C}^{m} \backslash\{0\} \times V_{y}\right) / / H^{\mathbb{C}}$ and two distinct $H^{\mathbb{C}}$-orbits in $\left(F \times \Phi_{y}\right)\left(U_{y}\right)$ may be separated by disjoint, $H^{\mathbb{C}}$-invariant neighborhoods.

Proof. We will omit the subscript and write $\Phi=\Phi_{y}$. We begin by showing that the map $(F \times \Phi): H^{\mathbb{C}} y \rightarrow \mathbb{C}^{m} \backslash\{0\} \times V$ is an embedding.

Let $w_{n} \in \mathbb{C}^{*}, h_{n} \in G^{\mathbb{C}}$ such that $(F \times \Phi)\left(w_{n} h_{n} y\right)$ converges in $\mathbb{C}^{m} \backslash\{0\} \times V$. We get $F\left(w_{n} h_{n} y\right)=F\left(w_{n} y\right)=\bigoplus w_{n}^{d_{i}} F_{i}(y)$, which converges against a non-zero 
value. There exists an $i$ such that $F_{i}(y) \neq 0$, hence $w_{n}$ is bounded from below and above, therefore we may assume that it converges in $\mathbb{C}^{*}$.

But then $w_{n}^{-1} \Phi\left(w_{n} h_{n} y\right)=\Phi\left(h_{n} y\right)$ converges and since $\Phi$ is an embedding on $G^{\mathbb{C}} y$, we have proved the claim.

We get that the $\mathbb{C}^{*}$-action on $\mathbb{C}^{m} \backslash\{0\}$ induced by the orders of the $F_{i}$ is proper and locally free, therefore we find a complex slice $S$ around $F(y)$ which is biholomorphic to some complex ball. Let $\Gamma=\mathbb{C}_{F(y)}^{*}$, then $\Omega:=\mathbb{C}^{*} \times{ }^{\Gamma} S$ is an open, $\mathbb{C}^{*}$-invariant Stein neighborhood of $F(y)$ in $\mathbb{C}^{m} \backslash\{0\}$ and the $H^{\mathbb{C}}$-orbit through $(F \times \Phi)(y)$ is closed in $\Omega \times V$.

Using [9, Einbettungssatz 1], we find a closed $H^{\mathbb{C}}$-equivariant embedding $\mu$ of $\Omega \times V$ into some $H^{\mathbb{C}}$-representation $V_{0}$. Now $\mu \circ(F \times \Phi)$, defined on $(F \times$ $\Phi)^{-1}(\Omega \times V)$, is an immersion in $y$ and an embedding on the $H^{\mathbb{C}}$-orbit through $y$.

From the proof of Proposition 5.1 in [22], one finds a slice $S_{0}$ through $\mu \circ(F \times$ $\Phi)(y)$ in $V_{0}$ and a slice $S_{Y}$ through $y$ in $Y$ such that the maps $\mu \circ(F, \Phi): S_{Y} \rightarrow S_{0}$ and also $\mu \circ(F \times \Phi): H^{\mathbb{C}} \times{ }^{L^{\mathbb{C}}} S_{Y} \rightarrow H^{\mathbb{C}} \times{ }^{L^{\mathbb{C}}} S_{0}$ are embeddings. Additionally, $H^{\mathbb{C}} \cdot S_{0}$ is saturated with respect to the quotient map.

Define $W_{0}=H^{\mathbb{C}} \cdot S_{0}, W:=\mu^{-1}\left(W_{0}\right)$ and $U:=H^{\mathbb{C}} \cdot S_{Y}$. Then $W$ is saturated with respect to the quotient map and $(F \times \Phi): U \rightarrow W$ is an embedding.

Now every $H^{\mathbb{C}}$-orbit in $U$ is closed, hence every $H^{\mathbb{C}}$-orbit in $\mu \circ(F \times \Phi)(U)$ is closed in $W_{0}$. We may separate two distinct, closed orbits in $W_{0}$ by $H^{\mathbb{C}}$-invariant open neighborhoods and the result follows.

Let us shortly summarize the assumptions we made to this point. We have that $H$ is a subgroup of its universal complexification $H^{\mathbb{C}}, G$ is a closed, normal subgroup of $H$ such that $H=G \rtimes S^{1}$. Furthermore, we assume $H^{\mathbb{C}}$ to be complex reductive. Now let $X$ be a CR manifold with proper, transversal CR action of $H$ such that $H_{x}^{0} \subset G_{x}^{0}$ for every $x \in X$ and $Y$ the universal equivariant extension of $X$. Our main result is the following.

Theorem 3.5. Let $X$ be as above and assume that $X / G$ is compact.

Then there exists a $H^{\mathbb{C}}$-representation $V$ and a $H^{\mathbb{C}}$-equivariant holomorphic embedding $\Phi: Y \rightarrow \mathbb{C}^{m} \backslash\{0\} \times V$, such that $\left.\Phi\right|_{X}: X \rightarrow \mathbb{C}^{m} \times V$ is a CR embedding. Here, $\mathbb{C}^{m}$ is the trivial $G^{\mathbb{C}}$-representation and decomposes into irreducible $\mathbb{C}^{*}$-representations with positive weights.

Proof. Let $F, \Phi_{y}, U_{y} \subset \Omega_{y}^{\mathbb{C}}$ and $W_{y}$ be as in Lemma 3.4.

Using that $X / G$ is compact, we may cover $Y$ be finitely many open subsets $U_{y}$ with $y \in I$ and consider

$$
\tilde{\Phi}:=\left(F, \bigoplus_{y \in I} \Phi_{y}\right): Y \rightarrow \mathbb{C}^{m} \times \bigoplus_{y \in I} V_{y},
$$

which is an immersion on $Y$ and injective on every $H^{\mathbb{C}}$-orbit.

We write $\tilde{V}:=\mathbb{C}^{m} \times \bigoplus_{y} V_{y}, \tilde{V}_{0}:=\left(\mathbb{C}^{m} \backslash\{0\}\right) \times \bigoplus_{y} V_{y}$ and show that the map $\tilde{\Phi}: Y \rightarrow \tilde{V}_{0}$ is proper. 
Let $a_{n}$ be a sequence in $Y$ such that $\tilde{\Phi}\left(a_{n}\right)=b_{n}$ converges against $b \in \tilde{V}_{0}$. Because $Y / H^{\mathbb{C}}=X / H$ is compact, we may assume that $\pi\left(a_{n}\right) \rightarrow \pi(a)$ converges, where we write $\pi: Y \rightarrow Y / H^{\mathbb{C}}$ for the quotient map.

Around $a$, we find a neighborhood $U_{y}$ and an open neighborhood $\tilde{W}_{y}$ of $\tilde{\Phi}(a)$, such that $\tilde{\Phi}: U_{y} \rightarrow \tilde{W}_{y}$ is an embedding and $\tilde{W}_{y}$ defines an open neighborhood of $b$ in $\tilde{V}_{0} / / H^{\mathbb{C}}$. This implies the convergence of a subsequence of $a_{n}$. Since $\inf _{x \in X}\|F(x)\|^{2}=c>0$, we conclude that $\tilde{\Phi}: X \rightarrow \tilde{V}$ is proper.

Since $\tilde{\Phi}$ is injective on every $H^{\mathbb{C}}$-orbit it only remains to show that we may separate points on different orbits.

Lemma 3.4 states that for every $w, z \in U_{y}$ in different orbits, we may separate $\tilde{\Phi}(w)$ and $\tilde{\Phi}(z)$ by $H^{\mathbb{C}}$-invariant open sets. For two distinct points $z, w \in Y$ in different $H^{\mathbb{C}}$-orbits, we may separate $z$ and $w$ by $H^{\mathbb{C}}$-invariant sets of the form $\Omega_{z}=H^{\mathbb{C}} \times{ }^{\mathbb{C}} S_{C}^{z}$ and $\Omega_{w}=H^{\mathbb{C}} \times{ }^{H_{w}^{\mathbb{C}}} S_{C}^{w}$. For every $d$ that is a multiple of the orders of $\mathbb{C}^{*}$ in $z$ and $w$, the map $f_{d}[h, z] \mapsto h_{C}^{d}$ is well-defined on $\Omega_{z} \cup \Omega_{w}$. Let $\varphi: \Omega_{z} \cup \Omega_{w} \rightarrow \mathbb{C}^{2}$ be equal to $(1,0)$ on $\Omega_{z}$ and equal to $(0,1)$ on $\Omega_{w}$. Note that $\varphi$ is equivariant with respect to the trivial $H^{\mathbb{C}}$-representation.

Applying Corollary 3.2 on the sheaf (6) for the map $f_{d} \cdot \varphi$ and sufficiently large $d$, we find a $G^{\mathbb{C}}$-invariant map $F_{z, w}: Y \rightarrow \mathbb{C}^{2}$ of order $d>0$ such that $F(z)$ and $F(w)$ may be separated by open, $H^{\mathbb{C}}$-invariant sets.

Because $\left(Y / H^{\mathbb{C}} \times Y / H^{\mathbb{C}}\right) / \bigcup_{y \in I}\left(U_{y} / H^{\mathbb{C}} \times U_{y} / H^{\mathbb{C}}\right)$ is compact, we conclude that we may use this argument finitely many times and the result follows.

\section{Line bundles}

We apply the methods used in the previous sections for a proof of an equivariant embedding theorem similar to the Kodaira embedding theorem.

Let $X$ be a compact CR manifold and $K$ a compact Lie group with transversal CR action on $X$.

We will use the definitions from [13] for rigid, positive CR line bundles, generalized to arbitrary compact groups.

Let $X$ be a CR manifold with transversal $K$-action. We say that a CR line bundle $L \rightarrow X$ is $K$-invariant if there exists a cover $U_{i}$ of $X$ over which $L$ is trivial such that each $U_{i}$ is $K$-invariant and the transition functions $g_{i j}$ are $K$-invariant and $\mathrm{CR}$. The transition functions can be viewed as holomorphic functions on $X / K$ and give rise to a holomorphic line bundle $L_{K} \rightarrow X / K$. On the other hand, every holomorphic line bundle $L_{K} \rightarrow X / K$ also induces a CR line bundle $L \rightarrow X$. We say that $L$ is positive if $L_{K}$ is positive.

Note that if $\Omega$ is a slice-neighborhood of $x$ in $X$ and $f: \Omega \rightarrow \mathbb{R}$ is a $K$-invariant smooth map, then $f$ extends to a $K^{\mathbb{C}}$-invariant map $F$ on $\Omega^{\mathbb{C}}$. Assume now that the hermitian form $(V, W) \mapsto i \partial \bar{\partial} F(V, \bar{W})$ is positive for $V, W \in T_{x}^{1,0} X$. We find a slice $S_{C}$ through $x$ in $\Omega^{\mathbb{C}}$ such that $\mathbb{C} T_{x} S_{C}=T_{x}^{1,0} X \oplus T_{x}^{0,1} X$, hence $\left.F\right|_{S_{C}}$ is strictly plurisubharmonic after possibly shrinking $S_{C}$. Since $\left(K_{x}\right)^{\mathbb{C}}$ acts as a finite group on $S_{C}$, we conclude that $\left.F\right|_{S_{C}}$ defines a strictly plurisubharmonic function 
on $S_{C} /\left(K_{x}\right)^{\mathbb{C}}$, using the same argument as at the end of Theorem 2.3. From [14, Proposition 6.1], we then see that if $L$ is a positive, invariant CR line bundle in the sense of [13], then it is also positive using the definition given above.

Note that every negative line bundle with metric $h$ is weakly negative. This means that the set $\{z \in L \mid h(z)<1\}$ is strongly pseudoconvex, see [7, 3 Satz 1].

Now let $L \rightarrow X$ be a weakly negative line bundle and $X_{S}$ the $S^{1}$-bundle of $L$, which is a strongly pseudoconvex CR manifold. Then $\mathbb{C} \times{ }^{1} X_{S} \cong L$ and $\mathbb{C} \times{ }^{1} X_{S} / K \cong L_{K}$.

If $V$ is a $K^{\mathbb{C}}$-representation and $\varphi: L \rightarrow V$ a CR map of order $d$, then we may define a section $X \rightarrow L^{-d} \otimes V$ by setting $s_{\varphi}(x):=1 \otimes \varphi(1, x)$ in a trivialization of $L$. A direct computation shows that this does indeed define a global section.

Now let $x \in X, \varphi: U \rightarrow V$ an equivariant injective immersion in some neighborhood $U$ into a $K^{\mathbb{C}}$-representation $V$ (see proof of theorem 3.3). Denote by $p: L \rightarrow X$ the bundle map. After shrinking $U$, we may assume that $L$ is trivial over $U$ and define the map $f_{d}: p^{-1}(U) \rightarrow \mathbb{C},(w, x) \mapsto w^{d}$. Applying Corollary 3.2 to the sheaf (5) for the point $(1,[x]) \in L_{K}$, the map $f_{d} \cdot(\varphi \circ p)$ and large $d$ gives an equivariant map $\Phi: L \rightarrow V_{d}$ with $\Phi=f_{d} \cdot(\varphi \circ p)$ of order 2 on $\left(S^{1} \times K\right) \cdot(1, x) \in L$.

The map $\Phi$ induces a section $\varphi_{0}: X \rightarrow L^{-d} \otimes V$. Indentifying $L^{-d} \otimes V$ with $V$ on $U$ using $c \otimes v \mapsto c v$, we get $\varphi_{0}(x)=\Phi(1, x)=f(1, x) \cdot(\varphi \circ p)(1, x)=\varphi(x)$. Let $v \in T_{x} U$, then $d_{x} \varphi_{0}(v)=d_{(1, x)} \Phi(0, v)=d_{(1, x)}(f \cdot(\varphi \circ p))(0, v)=d_{x} \varphi(v)$.

Denote by $\mathcal{L}^{k}(C)$ the $\mathrm{CR}$ sections $X \rightarrow L^{-d}$ and by $\mathcal{L}_{x}^{k}(X)$ and $\mathcal{L}_{x, y}^{k}(X)$ the sections vanishing in $x$ of order 2 and the sections vanishing in $x$ and $y$, respectively. For $x \in X$, we may use the argument above and choose a basis for $V$ to obtain sections $s_{1}, \ldots, s_{m} \in \mathcal{L}^{d}(X)$ such that the $d_{x} s_{i}$ form a generating system for $\left(T_{x} X\right)^{*}$. Using the same argument as above for $\varphi$ being a constant map, we also find a section $s \in \mathcal{L}^{d}(X)$ with $s(x) \neq 0$ and $d s(x)=0$. Using an analogous argument for Corollary 3.2 and sheaf (6), we also conclude that given two points $x, y \in X$ in different $K$-orbits, we find a section $s \in \mathcal{L}^{d_{x, y}}(X)$ such that $s(x)=0$ and $s(y) \neq 0$.

We have therefore shown that for every $x \in X$, there exists a $k$ such that the sequence

$$
0 \rightarrow \mathcal{L}_{x}^{k}(X) \rightarrow \mathcal{L}^{k}(X) \rightarrow\left(\mathcal{L}^{k} / \mathcal{L}_{x}^{k}\right)(X) \rightarrow 0
$$

is exact. Also, given $x, y \in X$, there exists a $k$ such that the sequence

$$
0 \rightarrow \mathcal{L}_{x, y}^{k}(X) \rightarrow \mathcal{L}^{k}(X) \rightarrow\left(\mathcal{L}^{k} / \mathcal{L}_{x, y}^{k}\right)(X) \rightarrow 0
$$

is exact.

Now if Sequence (7) is exact in $x$ for $k$, it is also exact in a neighborhood of $x$. The same applies for Sequence (8). The natural map $\Gamma\left(X,\left(L^{-d}\right)^{k}\right) \rightarrow \Gamma\left(X, L^{-d k}\right)$ is surjective, hence if sequences (7) and (8) are exact for $k$, they are also exact for $d k$ for every $d>0$.

Since $X$ is compact, we conclude that there exists a $k$ such that sequences (7) and (8) are exact for every point in $X$ and pairs of points in $X$, respectively. 
Corollary 4.1. Let $X$ be a compact $C R$ manifold with a transversal CR action of a compact Lie group $K$.

Assume that there exists a weakly negative line bundle $L_{K} \rightarrow X / K$. Then there exists a natural number $k$ and finitely many $C R$ sections $s_{i} \in \mathcal{L}^{k}(X)$ such that, for $W=\operatorname{span}\left(s_{i}\right)$, we have that

$$
\begin{array}{r}
X \rightarrow \mathbb{P}\left(W^{*}\right) \\
y \mapsto[s \mapsto s(y)]
\end{array}
$$

is a CR embedding.

Proof. For a subspace $W \subset \mathcal{L}^{k}(X)$, we denote by $\Phi_{W}$ the map defined in the corollary for this subspace. We may assume that $\Phi_{W}$ is always well-defined.

Choose $k$ so that Sequences (7) and (8) are exact for every $x \in X$ and pairs $x, y \in X$.

Take $x \in X$, then we find sections $g_{1}, \ldots, g_{m} \in \mathcal{L}^{k}$ such that $y \mapsto$ $\left(g_{1}(y), \ldots, g_{m}(y)\right)$ is an immersion in $x$ in a trivialization around $x$. We also get a section $g_{0}$ with $g_{0}(x) \neq 0$ and $d_{x} g_{0}=0$.

Set $W_{x}=\operatorname{span}\left(g_{0}, \ldots, g_{m}\right)$. We may assume that the $g_{i}$ form a basis for $W_{x}$, then $\Phi_{W_{x}}(y)=\left[g_{0}(y), \ldots, g_{m}(y)\right]$ in the corresponding dual basis. This shows that $\Phi_{W_{x}}$ is an immersion in $x$.

We may repeat this process finitely many times to find a subspace $W$ of $\mathcal{L}^{k}(X)$ such that $\Phi_{W}$ is an immersion on $X$. We may therefore cover $X$ by finitely many open sets $U_{i}$ such that $\Phi_{W}: U_{i} \rightarrow \mathbb{P}\left(W^{*}\right)$ is injective.

Now $(X \times X) / \bigcup_{i}\left(U_{i} \times U_{i}\right)$ is compact, and for two points $(x, y) \in(X \times$ $X) / \bigcup_{i}\left(U_{i} \times U_{i}\right)$, we find a section $\hat{s}$ such that $\Phi_{W+\mathbb{C} \hat{s}}$ separates $x$ and $y$.

We only need to do this finitely many times to ensure that $\Phi$ is injective.

Since the bundle is $K$-invariant, we have a $K$-action on the space of global sections defined by $k s(x):=s\left(k^{-1} x\right)$. We then get a $K$-action on $\Gamma(X, L)^{*}$ via $k \lambda(s):=\lambda\left(k^{-1} s\right)$. The map in Corollary 4.1 is equivariant if $W$ is $K$-invariant.

We may define a seminorm on the global CR sections by taking a $K$-invariant open subset $U$ of $X$ over which $L$ is trivial and defining $\|s\|_{U}:=\sup _{x \in U}\|s(x)\|^{2}+$ $\sup _{x \in U}\|d s(x)\|^{2}$. Since $X$ is compact, this induces a norm by taking the supremum over finitely many such seminorms. Then the $K$-action defined above is continuous. From [3, Proposition 3.6], we conclude that the $K$-finite sections are dense and the embedding from above can be chosen to be equivariant.

Open Access This article is licensed under a Creative Commons Attribution 4.0 International License, which permits use, sharing, adaptation, distribution and reproduction in any medium or format, as long as you give appropriate credit to the original author(s) and the source, provide a link to the Creative Commons licence, and indicate if changes were made. The images or other third party material in this article are included in the article's Creative Commons licence, unless indicated otherwise in a credit line to the material. If material is not included in the article's Creative Commons licence and your intended use is not permitted by statutory regulation or exceeds the permitted use, you will need to obtain permission directly 
from the copyright holder. To view a copy of this licence, visit http://creativecommons.org/ licenses/by/4.0/.

Funding Open Access funding enabled and organized by Projekt DEAL. Kevin Fritsch and Peter Heinzner were supported by the CRC TRR 191: "Symplectic Structures in Geometry, Algebra and Dynamics".

\section{References}

[1] Andreotti, A., Fredricks, G.A.: Embeddability of real analytic Cauchy-Riemann manifolds. Ann. Sc. Norm. Super. Pisa Cl. Sci. 6(2), 285-304 (1979)

[2] Baouendi, M.S., Rothschild, L.P., Treves, F.: CR structures with group action and extendability of CR functions. Invent. Math. 82, 359-396 (1985)

[3] Borel, A.: Représentations de groupes localement compacts. Springer, Berlin (1972)

[4] Boutet de Monvel, L.: Intégration des équations de Cauchy-Riemann induites formelles, Sémin. Équ. Dériv. Partielles (9), 1-13 (1974-1975)

[5] Fornaess, J., Narasimhan, R.: The Levi problem on complex spaces with singularities. Math. Ann. 248, 47-72 (1980)

[6] Grauert, H.: On Levi's problem and the imbedding of real-analytic manifolds. Ann. Math. 68(2), 460-472 (1958)

[7] Grauert, H.: Über Modifikationen und exzeptionelle analytische Mengen. Math. Ann. 146, 331-368 (1962)

[8] Grauert, H., Remmert, R.: Plurisubharmonische Funktionen in komplexen Räumen. Math. Z. 65, 175-194 (1956)

[9] Heinzner, P.: Linear äquivariante Einbettungen Steinscher Räume. Math. Ann. 280, $147-160$ (1988)

[10] Heinzner, P.: Geometric invariant theory on Stein spaces. Math. Ann. 289, 631-662 (1991)

[11] Heinzner, P.: Equivariant holomorphic extensions of real analytic manifolds. Bull. Soc. Math. France 121(3), 445-463 (1993)

[12] Herrmann, H., Hsiao, C.-Y., Li, X.: Szegö kernel expansion and equivariant embedding of CR manifolds with circle action. Ann. Glob. Anal. Geom. 52, 313-340 (2017)

[13] Hsiao, C.-Y., Li, X., Marinescu, G.: Equivariant Kodaira embedding of CR manifolds with circle action. Mich. Math. J. 70(1), 55-113 (2021)

[14] Hsiao, C.-Y., Marinescu, G.: Szegä kernel asymptotics and Morse inequalities on CR manifolds. Math. Z. 271, 509-553 (2012)

[15] Kohn, J.J.: The range of the tangential Cauchy-Riemann operator. Duke Math. J. 53(2), $525-545$ (1986)

[16] Lempert, L.: On three-dimensional Cauchy-Riemann manifolds. J. Am. Math. Soc. 5(4), 923-969 (1992)

[17] Loose, F.: A remark on the reduction of Cauchy-Riemann manifolds. Math. Nachr. 214, 39-51 (2000)

[18] Matsushima, Y.: Espaces homogènes de Stein des groupes de Lie complexes. Nagoya Math. J. 16, 205-218 (1960)

[19] Montgomery, D., Zippin, L.: Topological Transformation Groups. Dover publications, New York (1955)

[20] Palais, R.: On the existence of slices for actions of non-compact Lie groups. Ann. Math. 73(2), 295-323 (1961)

[21] Roberts, M.: A note on coherent G-sheaves. Math. Ann. 275, 573-582 (1986)

[22] Snow, D.M.: Reductive group actions on Stein spaces. Math. Ann. 259, $79-97$ (1982) 
Publisher's Note Springer Nature remains neutral with regard to jurisdictional claims in published maps and institutional affiliations. 